\title{
Semialgebraic Description of Teichmüller Space
}

\author{
By
}

\author{
Yohei KOMORI*
}

\begin{abstract}
We give a concrete semialgebraic description of Teichmüller space $T_{g}$ of the closed surface group $\Gamma_{g}$ of genus $g(\geq 2)$. Our result implies that for any $S L_{2}(\mathbf{R})$-representation of $\Gamma_{g}$, we can determine whether this representation is discrete and faithful or not by using $4 g-6$ explicit trace inequalities. We also show the connectivity and contractibility of $T_{g}$ from the point of view of $S L_{2}(\mathbf{R})$-representations of $\Gamma_{g}$. Previously, these properties of $T_{g}$ had been proved by using hyperbolic geometry and quasi-conformal deformations of Fuchsian groups. Our method is simple and only uses topological properties of the space of $S L_{2}(\mathbf{R})$-representations of $\Gamma_{g}$.
\end{abstract}

\section{Table of Contents}

\section{$\S 1$. Introduction}

§2. Construction of Teichmüller Space as a Semialgebraic Set

$\S 2.1$ The Space of $S L_{2}(\mathbf{R})$-Representations of the Surface Group $\Gamma$

$\S 2.2$ The Action of $P G L_{2}(\mathbf{R})$ on $R^{\prime}(\Gamma)$

$\S 2.3$ The Space of Characters of $\Gamma$

$\S 2.4$ The Relation Between $S L_{2}(\mathbf{R})$ - and $P S L_{2}(\mathbf{R})$-Representations of $\Gamma$

§3. Semialgebraic Description of Teichmüller Space $T_{g}(g=2)$

$\S 3.1$ Definition of the Semialgebraic Subset $S(\Gamma)$ of $X(\Gamma)$

$\$ 3.2$ Topological Structure of $S(\Gamma)$

$\S 3.3$ Cell Structure of Teichmüller Space $T_{2}$

$\S 3.4$ Semialgebraic Structure of Teichmüller Space $T_{2}$

§4. Semialgebraic Description of Teichmüller Space $T_{g}(g \geq 3)$

Communicated by K. Saito, December 12, 1995.

1991 Mathematics Subject Classification(s): 14L30, 30F60, 32G15

* Department of Mathematics

Osaka City University, Osaka 558, Japan 
$\S 4.1$ Definition of the Semialgebraic Subset $S(\mathbb{T})$ of $X(\mathbb{T})$

$\$ 4.2$ Topological Structure of $S(\mathbb{\Gamma})$

$\$ 4.3$ Cell Structure of Teichmüller Space $T_{g}$

$\S 4.4$ Semialgebraic Structure of Teichmüller Space $T_{g}$

\section{$\S 1 . \quad$ Introduction}

The Teichmüller space $T_{g}$ of compact Riemann surfaces of genus $g(\geq 2)$ is the moduli space of marked Riemann surfaces of genus $g$. Thanks to the uniformization theorem due to Klein, Koebe and Poincaré, any compact Riemann surface of genus $g(\geq 2)$ can be obtained as the quotient space $G \backslash \mathbb{H}$ where $\mathbb{H}$ is the upper half plane and $G$ is a cocompact Fuchsian group, i.e. a cocompact discrete subgroup of $P S L_{2}(\mathbb{R})$. As an abstract group, $G$ is isomorphic to the surface group $\Gamma_{g}$, which has the following presentation

$$
\Gamma_{g}:=\left\langle\alpha_{1}, \beta_{1}, \cdots, \alpha_{g}, \beta_{g} \mid \prod_{i=1}^{g}\left(\alpha_{i} \cdot \beta_{i} \cdot \alpha_{i}^{-1} \cdot \beta_{i}^{-1}\right)=i d .\right\rangle
$$

From this point of view, $T_{g}$ can be considered as the deformation space of a Fuchsian group which is isomorphic to $\Gamma_{g}$ and this is called Fricke space studied by Fricke himself and more precisely by $\mathbb{K}$ een $([\mathbb{F}],[\mathbb{K} 1],[\mathbb{K} 2]$, $[\mathrm{K} 3])$.

In this article, we consider this Fricke space from the point of view of $S L_{2}(\mathbb{R})$-representations of the surface group $\Gamma_{g}$. We treat $T_{g}$ as the $P G L_{2}(\mathbb{R})$-adjoint quotient of the set of discrete and faithful $\operatorname{PSL}_{2}(\mathbb{R})$ representations of $\mathbb{\Gamma}_{g}$

$$
T_{g}=\left\{\Gamma_{g} \rightarrow P S L_{2}(\mathbb{R}): \text { discrete and faithful }\right\} / P G L_{2}(\mathbb{R})
$$

where a discrete and faithful $P S L_{2}(\mathbb{R})$-representation of $\mathbb{\Gamma}_{g}$ means a group homomorphism from $\Gamma_{g}$ to $P S L_{2}(\mathbb{R})$ which is injective and the image of $\Gamma_{g}$ is a discrete subgroup of $P S L_{2}(\mathbb{R})$. Because any Fuchsian group which is isomorphic to $\mathbb{\Gamma}_{g}$ can be lifted to $S L_{2}(\mathbb{R})$ ([Pa], $[\mathrm{S}-\mathrm{S}]$ ), we can start from $\operatorname{Hom}\left(\Gamma_{g}, S L_{2}(\mathbb{R})\right)$ the set of $S L_{2}(\mathbb{R})$-representations of $\Gamma_{g}$. And $T_{g}$ can be considered as the set of characters of discrete and faithful $S L_{2}(\mathbb{R})$-representations of $\Gamma_{g}$.

From this point of view, we can get a real algebraic structure on $T_{g}$ as follows. By using the presentation of $\Gamma_{g}, \operatorname{Hom}\left(\Gamma_{g}, S L_{2}(\mathbb{R})\right)$ can be embeded 
into the product space $S L_{2}(\mathbb{R})^{2 g}$ as the real algebraic subset $R(\Gamma)$ which is called the space of representations ([C-S], [Go], [M-S ]). The adjoint action of $P G L_{2}(\mathbb{R})$ on $R(\Gamma)$ induces the action on $\mathbb{R}[R(\Gamma)]$ the affine coordinate ring of $R(\Gamma)$ and put $\mathbb{R}[R(\Gamma)]^{P G L_{2}(\mathbb{R})}$ the ring of invariants under this action. Let $X(\Gamma)$ be a real algebraic set whose affine coordinate ring is isomorphic to $\mathbb{R}[R(\Gamma)]^{P G L_{2}(\mathbf{R})}$. Then $T_{g}$ can be realized as a semialgebraic subset of $X(\Gamma)$. Hence $T_{g}$ is determined by finitely many polynomial equalities and inequalities on $X(\Gamma)$. This construction is essentially due to Helling [He], and later Culler-Shalen [C-S] and Morgan-Shalen [M-S] made this process more clear. And by using this procedure, Brumfiel described the real spectrum compactification of $T_{g}[\mathrm{Br}$ ]. More recently, in a series of works [Sa 1], [Sa 2] and [Sa 3], Saito obtained a description of the coordinate ring for $T_{g}$ as a semialgebraic set defined over $\mathbb{Z}$.

Our theme in this paper is to study the semialgebraic structure on $T_{g}$, and we mainly consider the following two things. First we describe the defining equations of $T_{g}$ on $X(\Gamma)$ by using $6 \mathrm{~g}-6$ explicit polynomial inequalities. (Theorems 3.2 and 4.2). This problem is related to the construction of the global coordinates of $T_{g}$ by means of a small number of traces of elements of Fuchsian groups which have been studied deeply by Keen ([K1], [K2], [K3]) and more recently by Okai and Okumura ([Ok], [O1], [O2]) by using hyperbolic geometry on $\mathbb{H}$ and an argument involving the fundamental polygons of Fuchsian groups. Our treatment in this paper is rather algebraic. The second is that from a real algebraic viewpoint, we also show the well known fact that $T_{g}$ is a $6 \mathrm{~g}-6$ dimensional cell (Theorems 3.1 and 4.1) which was proved by Teichmüller himself by means of his theory of quadratic differentials and quasi-conformal mappings.

For our purposes, we only need some elementary topological properties of semialgebraic sets and some geometric properties of the space of representations $R(\Gamma)$. More precisely, the next three assertions are essential for our arguments (the following notations are defined in Sections 3 and 4):

1. Let $R_{0}(\Gamma)$ be the set of discrete and faithful $S L_{2}(\mathbb{R})$-repretations of $\Gamma_{g}$. Then $R_{0}(\Gamma)$ consists of finitely many connected components of $R(\Gamma)=\operatorname{Hom}\left(\Gamma_{g}, S L_{2}(\mathbb{R})\right)$.

2. Let $t^{-1}(S(\Gamma))$ be the of $S L_{2}(\mathbb{R})$-representations $\rho$ of $\Gamma_{g}$ satisfying the following $2 \mathrm{~g}-3$ trace inequalities 


$$
\begin{aligned}
\operatorname{tr}\left(\rho\left(\left[\alpha_{i}, \beta_{i}\right]\right)\right) & <-2(i=1, \cdots, g) \\
\operatorname{tr}\left(\rho\left(\left[\alpha_{1}, \beta_{1}\right] \cdots\left[\alpha_{j}, \beta_{j}\right]\right)\right) & <-2(j=1, \cdots, g-2)
\end{aligned}
$$

where $[\alpha, \beta]:=\alpha \cdot \beta \cdot \alpha^{-1} \cdot \beta^{-1}$. Then $t^{-1}(S(\Gamma))$ consists of $2^{4 g-3} \times 2$ connected components such that each components is homemorphic to $\mathbb{R}^{6 g-6} \times P S L_{2}(\mathbb{R})$ and this homeomorphism is $P S L_{2}(\mathbb{R})$-equivariant.

3. $R_{0}(\Gamma) \subset t^{-1}(S(\Gamma))$.

In fact, we will construct global coordinates on $t^{-1}(S(\Gamma))$, and by using these coordinates, we shall find a system of trace inequalities which characterize $R_{0}(\Gamma)$ in $t^{-1}(S(\Pi))$. Finally, we obtain a semialgebraic description of $T_{g}$ and a cell structure of $T_{g}$. The trace inequalities which define $t^{-1}(S(\Gamma))$ in Assertion 2 above already appeared in the paper of Seppälä-Sorvali (S-S]), and in that paper, they used these inequalities to solve the lifting problem of a Fuchsian group to $S L_{2}(\mathbf{R})$. At first, I proved Assertion 2 above by using a slightly complicated method involving some geometric properties of $R(\Gamma)$, and II would like to thank Professor Kyoji Saito for telling me about the result of [S-S]. These inequalities also appeared in the paper of Okumura [O2].

The remainder of this paper is organized as follows. Section 2 deals with the construction of Teichmüller space $T_{g}$ following Culler-Shalen [C-S] and Morgan-Shalen [M-S]. The description of the defining inequalities and cell structure of $T_{g}$ are given in Sections 3 and 4 . In Section 3 we treat the case of genus $g=2$ and in Section 4, the case of genus $g \geq 3$ is discussed.

Finally I would like to express the deepest thanks to the following persons: Kyoji Saito my advisor who helped me constantly on the way to the completion of this work, and from whom I learned the excitement of doing mathematics; and Yoshikazu Yamagishi and Yasushi Yamashita for the interest that they expressed at every stage, as well as for lengthy discussions which were very meaningful for me. Most of the work contained in this paper was done at the Saturday Seminar at RIMS, Kyoto University, 1992-93, and I would also like to thank referees for their helpful comments and corrections.

\section{§2. Construction of Teichmüller Space as a Semialgebraic Set}

In this section we review the construction of Teichmüller space following [C-S], [M-S], [Sa 1], [Sa 2], [Sa 3]. 


\section{§2.1. The Space of $S L_{2}(\mathbf{R})$-Representations of the Surface Group $\Gamma$}

Let $g \geq 2$ be fixed. We define the (closed) surface group of genus $g$ by the following presentation

$$
\Gamma=\Gamma_{g}:=\left\langle\alpha_{1}, \beta_{1}, \cdots, \alpha_{g}, \beta_{g} \mid \prod_{i=1}^{g}\left[\alpha_{i}, \beta_{i}\right]=i d .\right\rangle
$$

where $\left[\alpha_{i}, \beta_{i}\right]:=\alpha_{i} \cdot \beta_{i} \cdot \alpha_{i}^{-1} \cdot \beta_{i}^{-1}$.

By using this presentation, we can embed $\operatorname{Hom}\left(\Gamma, S L_{2}(\mathbf{R})\right)$ the set of $S L_{2}(\mathbf{R})$-representations of $\Gamma$ into the product space $S L_{2}(\mathbf{R})^{2 g}$ and let $R(\Gamma)$ denote the image of $\operatorname{Hom}\left(\Gamma, S L_{2}(\mathbb{R})\right)$

$$
\begin{aligned}
\operatorname{Hom}\left(\Gamma, S L_{2}(\mathbf{R})\right) & \rightarrow R(\Gamma) \subset S L_{2}(\mathbf{R})^{2 g} \\
\rho & \mapsto\left(\rho\left(\alpha_{1}\right), \rho\left(\beta_{1}\right), \cdots, \rho\left(\alpha_{g}\right), \rho\left(\beta_{g}\right)\right) .
\end{aligned}
$$

We identify $R(\Gamma)$ and $\operatorname{Hom}\left(\Gamma, S L_{2}(\mathbb{R})\right)$. In the following we also identify a representation $\rho$ and the image $\left(A_{1}, B_{1}, \cdots, A_{g}, B_{g}\right) \in S L_{2}(\mathbb{R})^{2 g}$ of the system of generators $\left\{\alpha_{1}, \beta_{1}, \cdots, \alpha_{g}, \beta_{g}\right\}$ of $\Gamma$ under $\rho . R(\Gamma)$ is a real algebraic set and we call this the space of $S L_{2}(\mathbb{R})$-representations of $\Gamma . P G L_{2}(\mathbb{R})$ acts on $R(\Gamma)$ from the right-hand side

$$
\begin{aligned}
R(\Gamma) \times P G L_{2}(\mathbf{R}) & \rightarrow R(\Gamma) \\
(\rho, P) & \mapsto P^{-1} \rho P .
\end{aligned}
$$

We remark that although we use the system of generators $\left\{\alpha_{1}, \beta_{1}, \cdots, \alpha_{g}, \beta_{g}\right\}$ of $\Gamma$ to define $R(\Gamma)$, the real algebraic structure of $R(\Gamma)$ does not depend on this system of generators. In fact if we choose another system of generators of $\Gamma$ consisting of $N$ elements and embed $\operatorname{Hom}\left(\Gamma, S L_{2}(\mathbf{R})\right)$ into the product space $S L_{2}(\mathbf{R})^{N}$, we get another real algebraic set but it is canonically isomorphic to $R(\Gamma)$.

Next we consider the following subset of $R(\Gamma)$

$$
R^{\prime}(\Gamma):=\{\rho \in R(\Gamma) \mid \rho \text { is non abelian and irreducible }\}
$$

where a representation $\rho$ is non abelian if $\rho(\Gamma)$ is a non abelian subgroup of $S L_{2}(\mathbf{R})$ and $\rho$ is irreducible if the action of $\rho(\Gamma)$ on $\mathbf{R}^{2}$ admits no nontrivial invariant subspaces. Hence if $\rho$ is not irreducible (i.e., reducible) then there 
exists $P \in P G L_{2}(\mathbb{R})$ such that $P^{-1} \rho(\Gamma) P$ consists of upper triangular matrices, hence in particular $\rho(\Gamma)$ is solvable. We remark that the action of $P G L_{2}(\mathbb{R})$ on $R(\Gamma)$ preserves $R^{\prime}(\Gamma)$. The next lemma is useful for the study of $R^{\prime}(\Gamma)$.

Lemma 2.1. For $\rho \in R^{\prime}(\mathbb{\Gamma})$, there exist $g, h \in \Gamma$ such that $\rho(g)$ is a hyperbolic matrix, i e., $|\operatorname{tr}(\rho(g))|>2$, and $\rho(h)$ has no fixed points in common with $\rho(g)$. In other words there exists $P \in P G L_{2}(\mathbb{R})$ such that

$$
\begin{aligned}
& P^{-1} \rho(g) P=\left(\begin{array}{ll}
\lambda & 0 \\
0 & \frac{1}{\lambda}
\end{array}\right) \quad(\lambda \neq \pm 1) \\
& P^{-1} \rho(h) P=\left(\begin{array}{ll}
a & b \\
c & d
\end{array}\right) \quad(b \cdot c \neq 0) .
\end{aligned}
$$

Proof. For $\rho \in R^{\prime}(\Gamma)$, suppose that $\rho(\Gamma)$ has no hyperbolic elements. If there exists a parabolic element $\rho(g) \in \rho(\mathbb{\Gamma})$, there exists $P \in P G L_{2}(\mathbb{R})$ such that

$$
P^{-1} \rho(g) P= \pm\left(\begin{array}{ll}
1 & q \\
0 & 1
\end{array}\right) \quad(q \neq 0)
$$

As $\rho$ is irreducible, there exists $h \in \mathbb{\Gamma}$ with

$$
P^{-1} \rho(h) P=\left(\begin{array}{ll}
a & b \\
c & d
\end{array}\right) \quad(c \neq 0) .
$$

Then for $n \in \mathbb{N}$

$$
\operatorname{tr}\left(\rho(g)^{n} \cdot \rho(h)\right)=( \pm 1)^{n}(\operatorname{tr}(\rho(h))+n c q)
$$

and $\rho(g)^{n} \cdot \rho(h)=\rho\left(g^{n} h\right)$ is hyperbolic for sufficiently large $n$. This is a contradiction. Next if every element of $\rho(\Gamma)-\{i d\}$ is elliptic, as $\rho$ is non abelian, there exist $g, h \in \Gamma$ with $[\rho(g), \rho(h)] \neq\left(\begin{array}{ll}1 & 0 \\ 0 & 1\end{array}\right)$. Then there exists $P \in P G L_{2}(\mathbb{R})$ such that

$$
\begin{aligned}
& P^{-1} \rho(g) P=\left(\begin{array}{cc}
\cos \theta & -\sin \theta \\
\sin \theta & \cos \theta
\end{array}\right) \quad(\theta \neq 0, \pi) \\
& P^{-1} \rho(h) P=\left(\begin{array}{ll}
a & b \\
c & d
\end{array}\right)
\end{aligned}
$$


with $a^{2}+b^{2}+c^{2}+d^{2} \neq 2$, and $\operatorname{tr}(\rho([g, h]))=2+\sin ^{2} \theta\left(a^{2}+b^{2}+c^{2}+d^{2}-2\right)>2$. This is a contradiction. Hence there exists $g \in \Gamma$ so that $\rho(g)$ is hyperbolic and

$$
P^{-1} \rho(g) P=\left(\begin{array}{ll}
\lambda & 0 \\
0 & \frac{1}{\lambda}
\end{array}\right) \quad(\lambda \neq \pm 1)
$$

for some $P \in P G L_{2}(\mathbb{R})$. Next suppose that any element of $P^{-1} \rho(\Gamma) P$ is either upper or lower triangular matrix. Because $\rho$ is irreducible there exist $h_{1}, h_{2} \in \Gamma$ such that $P^{-1} \rho\left(h_{1}\right) P$ is an upper triangular matrix and $P^{-1} \rho\left(h_{2}\right) P$ is a lower triangular matrix. But $P^{-1} \rho\left(h_{1} h_{2}\right) P$ is not a triangular matrix. This is a contradiction. Therefore there exists $h \in \Gamma$ so that

$$
P^{-1} \rho(h) P=\left(\begin{array}{ll}
a & b \\
c & d
\end{array}\right) \quad(b \cdot c \neq 0) .
$$

We have another characterization of $R^{\prime}(\Gamma)$.

\section{Proposition 2.1.}

$$
\begin{aligned}
R^{\prime}(\Gamma) & =\{\rho \in R(\Gamma) \mid \operatorname{tr}(\rho([a, b])) \neq 2 \text { for some } a, b \in \Gamma\} \\
& =R(\Gamma)-\bigcap_{a, b \in \Gamma}\{\rho \in R(\Gamma) \mid \operatorname{tr}(\rho([a, b]))=2\}
\end{aligned}
$$

Proof. $(\Rightarrow)$ Take $g, h \in \Gamma$ which satisfy the conditions of Lemma 2.1. Then $\operatorname{tr}([\rho(g), \rho(h)]) \neq 2$.

$(\Leftrightarrow)$ If $\rho(\Gamma)$ is abelian, $[\rho(a), \rho(b)]=\left(\begin{array}{ll}1 & 0 \\ 0 & 1\end{array}\right)$ for any $a, b \in \Gamma$. If $\rho(\Gamma)$ has a non trivial invariant subspace, there exists $P \in P G L_{2}(\mathbb{R})$ such that any element of $P^{-1} \rho(\Gamma) P$ is an upper triangular matrix. Hence $\operatorname{tr}([\rho(a), \rho(b)])=2$ for any $a, b \in \Gamma$.

Corollary 2.1. $R^{\prime}(\Gamma)$ is open in $R(\Gamma)$.

Proposition 2.2. $R^{\prime}(\Gamma)$ has a structure of a $6 g-3$ dimensional real analytic manifold.

Proof. We consider the mapping $r$ 


$$
\begin{gathered}
r: S L_{2}(\mathbb{R})^{2 g} \rightarrow S L_{2}(\mathbb{R}) \\
\left(A_{1}, B_{1}, \cdots, A_{g}, B_{g}\right) \mapsto \prod_{i=1}^{g}\left[A_{i}, B_{i}\right] .
\end{gathered}
$$

Then $R(\Gamma)=r^{-1}\left(\left(\begin{array}{ll}1 & 0 \\ 0 & 1\end{array}\right)\right)$. We will check that the rank of the differential $(d r)_{\rho}$ at $\rho \in R^{\prime}(\Gamma)$ is maximal. By regarding $s l_{2}(\mathbb{R})$, the Lie algebra of $S L_{2}(\mathbb{R})$, as the Lie algebra of left-invariant vector fields on $S L_{2}(\mathbb{R}),(d r)_{\rho}$ can be written as follows. For $\rho=\left(A_{1}, B_{1}, \cdots, A_{g}, B_{g}\right) \in R^{\prime}(\Gamma)$

$$
\begin{aligned}
&(d r)_{\rho}: s l_{2}(\mathbb{R})^{2 g} \rightarrow s l_{2}(\mathbb{R}) \\
&\left(\xi_{i}, \eta_{i}\right)_{1 \leq i \leq g} \mapsto \sum_{i=1}^{g}\left\{\delta_{F_{1} A_{t} F_{1}^{-1}}\left(F_{i} \eta_{i} F_{i}^{-1}\right)-\delta_{F_{l} B_{t} F_{l}^{-1}}\left(F_{i} \xi_{i} F_{i}^{-1}\right)\right\}
\end{aligned}
$$

where $F_{i}:=\Pi_{l=1}^{i-1}\left[A_{l}, B_{l}\right] A_{i} B_{i}$ and for $A \in S L_{2}(\mathbb{R})$ and $X \in S l_{2}(\mathbb{R}), \delta_{A}(X):=$ $X-A^{-1} X A$.

Put $S_{i}:=F_{i} A_{i} F_{i}^{-1}, S_{i+g}:=F_{i} B_{i} F_{i}^{-1}(i=1, \cdots, g)$. Then $\left\{S_{k}\right\}$ is a system of generators of $\rho(\Gamma)$. As $\rho \in R^{\prime}(\Gamma)$, some $S_{j}$ is not the identity matrix hence $S_{j}$ is hyperbolic, parabolic or elliptic. First we assume that $S_{j}$ is hyperbolic. Then there exists $P \in P G L_{2}(\mathbb{R})$ such that

$$
S_{j}=P\left(\begin{array}{ll}
\lambda & 0 \\
0 & \frac{1}{\lambda}
\end{array}\right) P^{-1} \quad(\lambda \neq \pm 1)
$$

Because $\rho \in R^{\prime}(\Gamma)$, there exists $S_{k}$ such that

$$
S_{k}=P\left(\begin{array}{ll}
p & q \\
r & s
\end{array}\right) P^{-1} \quad(q \neq 0 \text { or } r \neq 0) \text {. }
$$

If we put $X_{j}=P\left(\begin{array}{cc}x_{j} & y_{j} \\ z_{j} & -x_{j}\end{array}\right) P^{-1}, X_{k}=P\left(\begin{array}{cc}x_{k} & y_{k} \\ z_{k} & -x_{k}\end{array}\right) P^{-1} \in S l_{2}(\mathbf{R})$

$$
\begin{aligned}
& \delta_{S_{j}}\left(X_{j}\right)=P\left(\begin{array}{cc}
0 & \left(1-\frac{1}{\lambda^{2}}\right) y_{j} \\
\left(1-\lambda^{2}\right) z_{j} & 0
\end{array}\right) P^{-1} \\
& \delta_{S_{k}}\left(X_{k}\right)=P\left(\begin{array}{cc}
(1-p s-q r) x_{k}+p q z_{k}-r s y_{k} & * \\
* & *
\end{array}\right) P^{-1} .
\end{aligned}
$$

Therefore the mapping 


$$
\begin{aligned}
s l_{2}(\mathbf{R}) \times s l_{2}(\mathbf{R}) & \rightarrow s l_{2}(\mathbf{R}) \\
\left(X_{j}, X_{k}\right) & \mapsto \delta_{S_{J}}\left(X_{j}\right)+\delta_{S_{k}}\left(X_{k}\right)
\end{aligned}
$$

is surjective, and this shows the surjectivity of $(d r)_{\rho}$. By a similar argument, one can also show the surjectivity of $(d r)_{\rho}$ for the case that $S_{j}$ is parabolic or elliptic. Hence by the implicit function theorem $R^{\prime}(\Gamma)$ has the structure of a $6 \mathrm{~g}-3$ dimensional real analytic manifold.

Next we define the subset $R_{0}(\Gamma)$ of $R(\Gamma)$ by

$$
R_{0}(\Gamma):=\{\rho \in R(\Gamma) \mid \rho \text { is discrete and faithful }\}
$$

where a representation $\rho$ is discrete if $\rho(\Gamma)$ is a discrete subgroup of $S L_{2}(\mathbb{R})$ and $\rho$ is faithful if $\rho$ is injective. We remark that the action of $P G L_{2}(\mathbf{R})$ on $R(\Gamma)$ preserves $R_{0}(\Gamma)$. Then another characterization of $R_{0}(\Gamma)$ is

\section{Proposition 2.3.}

$$
\begin{aligned}
R_{0}(\Gamma) & =\{\rho \in R(\Gamma) \mid \rho \text { is cocompact, discrete and faithful }\} \\
& =\{\rho \in R(\Gamma) \mid \rho \text { is purely hyperbolic }\}
\end{aligned}
$$

where a representation $p$ is cocompact if the quotient space $\rho(\Gamma) \backslash S L_{2}(\mathbf{R})$ is compact with respect to the quotient topology, and $\rho$ is called purely hyperbolic if $\rho(h)$ is hyperbolic for any $h(\neq$ identity $) \in \Gamma$.

Proof. (1) $\Rightarrow(2) \quad$ The fundamental group of a surface $\rho(\Gamma) \backslash \mathbf{H}$ is isomorphic to the surface group $\Gamma$. Hence $\rho(\Gamma) \backslash H$ is compact.

(2) $\Rightarrow$ (3) Because $\rho(\Gamma)$ is discrete, any elliptic element of $\rho(\Gamma)$ is finite order. But $\Gamma$ is torsion free. Thus $\rho(\Gamma)$ has no elliptic elements. Moreover if $\rho(\Gamma)$ has a parabolic element, then $\rho(\Gamma) \backslash \mathbf{H}$ has a cusp. Since $\rho(\Gamma) \backslash \mathbf{H}$ is compact, $\rho(\Gamma)$ has no parabolic elements.

(3) $\Rightarrow(1)$ Faithfulness is immediate. Discreteness follows from Nielsen's theorem (see [Si, Theorem 3, p33]).

Proposition 2.4. $R_{0}(\Gamma)$ is open and closed in $R(\Gamma)$.

Proof. We give a sketch of a proof. We recall the inequalities of Jørgensen ([Jø], especially the argument of Proposition 1, §3): 
For any $\rho \in R(\Gamma) \rho$ is contained in $R_{0}(\Gamma)$ if and only if

$$
|\operatorname{tr}([\rho(g), \rho(h)])-2|+\left|\operatorname{tr}(\rho(h))^{2}-4\right| \geq 1
$$

for any pair $g, h \in \Gamma$ with $g h \neq h g$.

These inequalities are closed conditions of $R_{0}(\Gamma)$ in $R(\Gamma)$.

The openness of $R_{0}(\Gamma) \subset \mathbb{R}(\Gamma)$ follows from the next theorem due to Weil [W]:

If $G$ is a coonnected Lie group and $\Gamma$ is a discrete group, then the set of cocompact, discrete and faithful representations from $\Gamma$ to $G$ is open in the set of all representations from $\Gamma$ to $G$.

Next we recall the notions of a semialgebraic set. Let $\mathrm{V}$ be a real algebraic set with affine coordinate ring $\mathbb{R}[\mathrm{V}]$, i.e., the ring of polynomial functions on V. A subset $\mathrm{S}$ of $\mathrm{V}$ is called a semialgebraic subset of $V$ if there exist finitely many polynomial functions $f_{i}, g_{i_{1}}, \cdots g_{i_{m(1)}}(i=1, \cdots, l)$ on $\mathrm{V}$ such that $\mathrm{S}$ can be written as

$$
S=\bigcup_{i=1}^{l}\left\{x \in V \mid f_{i}(x)=0, g_{i_{1}}(x)>0, \cdots g_{i_{m(1)}}(x)>0\right\} .
$$

From the above definition, it follows immediately that any real algebraic set is a semialgebraic set. Moreover, it is known that any connected component of a semialgebraic set (with respect to the Euclidean topology) is also a semialgebraic set and the number of connected components of a semialgebraic set is finite (see [B-C-R] Theorem 2.4.5).

Corollary 2.2. $R_{0}(\Gamma)$ consists of finitely many connected components of $R(\Gamma)$, hence $R_{0}(\Gamma)$ is a semialgebraic subset of $R(\Gamma)$.

The relation between $R^{\prime}(\Gamma)$ and $R_{0}(\Gamma)$ is

Proposition 2.5. $R_{0}(\Gamma) \subset R^{\prime}(\Gamma)$.

Proof. Let $\rho$ be a element of $R_{0}(\Gamma)$. Since the surface group $\Gamma$ is non abelian and $\rho$ is injective, $\rho$ is non abelian. Also beccause $\Gamma$ is not solvable, $\rho$ is irreducible. 
Corollary 2.3. $\quad R_{0}(\Gamma)$ has the structure of a $6 g-3$ dimensional real analytic manifold.

\section{§2.2. The Action of $P G L_{2}(\mathbb{R})$ on $R^{\prime}(\Gamma)$}

This subsection follows the argument of Gunning (Section 9 in [Gu]). We will show that the quotient space $R^{\prime}(\Gamma) / P G L_{2}(\mathbb{R})$ under the action defined in Subsection 2.1 has the structure of a $6 \mathrm{~g}-6$ dimensional real analytic manifold such that the natural projection

$$
R^{\prime}(\Gamma) \rightarrow R^{\prime}(\Gamma) / P G L_{2}(\mathbb{R})
$$

is a real analytic principal $P G L_{2}(\mathbb{R})$-bundle.

Lemma 2.2. $P G L_{2}(\mathbb{R})$ acts on $R^{\prime}(\Gamma)$ without fixed points.

Proof. For $P \in P G L_{2}(\mathbb{R})$ and $\rho \in R^{\prime}(\Gamma)$ suppose that $P^{-1} \rho P=\rho$. Then by Lemma 2.1 there exists $g \in \Gamma$ and $Q \in P G L_{2}(\mathbb{R})$ such that

$$
Q^{-1} \rho(g) Q=\left(\begin{array}{ll}
\alpha & 0 \\
0 & \frac{1}{\alpha}
\end{array}\right) \quad(\alpha \neq \pm 1) .
$$

Then $P^{-1} \rho P=\rho$ implies that $Q^{-1} P Q$ is also diagonal, and if $Q^{-1} P Q$ is not the identity, then $Q^{-1} \rho(h) Q$ is also diagonal for any $h \in \Gamma$, but this contradicts the fact that $\rho \in R^{\prime}(\Gamma)$. Therefore, $Q^{-1} P Q$, hence also $P$, is the identity.

Lemma 2.3. The action of $P G L_{2}(\mathbb{R})$ on $R^{\prime}(\Gamma)$ is proper: i.e., every element of $R^{\prime}(\Gamma)$ has an open neighborhood $U \subseteq R^{\prime}(\Gamma)$ such that the closure of the set

$$
\left\{P \in P G L_{2}(\mathbb{R}) \mid(U \cdot P) \cap U \neq \phi\right\}
$$

(where $U \cdot P:=\left\{P^{-1} \rho P \mid \rho \in U\right\}$ is compact in $P G L_{2}(\mathbb{R})$.

Proof. By Proposition $2.2 R^{\prime}(\Gamma)$ is a real analytic manifold. Hence any $\rho$ of $R^{\prime}(\Gamma)$ has an open neighborhood $U \subset R^{\prime}(\Gamma)$ such that $\bar{U}$ is compact in $R^{\prime}(\Gamma)$. Because $S O(2)$ is compact, by replacing $U$ by $U \cdot S O(2)$ if necessary, we may assume that $U$ is invariant under the adjoint action of $S O(2)$. Suppose that there is a sequence $\left\{T_{v}\right\} \subset P G L_{2}(\mathbb{R})$ such that $\left(U \cdot T_{v}\right) \cap U \neq \phi$ for each $v$ but $\left\{T_{v}\right\}$ has no accumulation points in $P G L_{2}(\mathbb{R})$. $T_{v}$ can be considered as 
an element of $S L_{2}(\mathbf{R})$ or $\left(\begin{array}{cc}-1 & 0 \\ 0 & 1\end{array}\right) S L_{2}(\mathbf{R})$. Then by taking a subsequence we may assume that $\left\{T_{v}\right\} \subset S L_{2}(\mathbf{R})$ or $\left\{T_{v}\right\} \subset\left(\begin{array}{cc}-1 & 0 \\ 0 & 1\end{array}\right) S L_{2}(\mathbf{R})$. First we treat the case that $\left\{T_{v}\right\} \subset S L_{2}(\mathbb{R})$ (a similar argument works for the other case). Then $T_{v}$ can be written as

$$
T_{v}=A_{v} \cdot B_{v}
$$

where $A_{v} \in S O(2)$ and $B_{v}=\left(\begin{array}{cc}a_{v} & b_{v} \\ 0 & \frac{1}{a_{v}}\end{array}\right)$. Because $\left\{T_{v}\right\}$ has no accumulation points in $P G L_{2}(\mathbf{R})$ we may assume that

$$
\left|a_{v}\right| \rightarrow 0, \quad\left|a_{v}\right| \rightarrow \infty \text { or }\left|b_{v}\right| \rightarrow \infty \quad(\text { as } v \rightarrow \infty) .
$$

Since $U$ is $S O(2)$-invariant

$$
\left(U \cdot B_{v}\right) \cap U \neq \phi .
$$

Hence there exists $\rho_{v} \in U$ so that $B_{v}^{-1} \rho_{v} B_{v} \in U$ for each $v$. Since $\bar{U}$ is compact we may assume that $\left\{\rho_{v}\right\}$ and $\left\{B_{v}^{-1} \rho_{v} B_{v}\right\}$ converge to $\rho \in \bar{U}$ and $\eta \in \bar{U}$ respectively.

For $g \in \Gamma$ put

$$
\begin{aligned}
\rho_{v}(g) & =\left(\begin{array}{ll}
p_{v} & q_{v} \\
r_{v} & s_{v}
\end{array}\right) \\
\rho(g) & =\lim _{v \rightarrow \infty} \rho_{v}(g)=\left(\begin{array}{ll}
p_{\infty} & q_{\infty} \\
r_{\infty} & s_{\infty}
\end{array}\right) \\
\eta(g) & =\lim _{v \rightarrow \infty} B_{v}^{-1} \rho_{v}(g) B_{v}=\left(\begin{array}{ll}
p_{\infty}^{\prime} & q_{\infty}^{\prime} \\
r_{\infty}^{\prime} & s_{\infty}^{\prime}
\end{array}\right) .
\end{aligned}
$$

Then

$$
B_{v}^{-1} \rho_{v} B_{v}=\left(\begin{array}{cc}
p_{v}-a_{v} b_{v} r_{v} & \frac{b_{v}}{a_{v}}\left(p_{v}-s_{v}\right)+\frac{1}{a_{v}^{2}} q_{v}-b_{v}^{2} r_{v} \\
a_{v}^{2} r_{v} & a_{v} b_{v} r_{v}+s_{v}
\end{array}\right)
$$

Since $\rho_{v}, \rho, \eta$ and $B_{v}^{-1} \rho_{v} B_{v}$ are all contained in the compact set $\bar{U}$, each component of the matrices of (4), (5), (6) and (7) is bounded. If $\left|a_{v}\right| \rightarrow \infty$, the boundedness of $\left\{a_{v}^{2} r_{v}\right\}$ implies that $r_{\infty}=\lim _{v \rightarrow \infty} r_{v}=0$. If $\left\{a_{v}\right\}$ is bounded and does not converge to 0 and $\left|b_{v}\right| \rightarrow \infty$, the boundedness of $\left\{a_{v} b_{v} r_{v}+s_{v}\right\}$ also implies $r_{\infty}=\lim _{v \rightarrow \infty} r_{v}=0$. In these cases, for any $g \in \Gamma, \rho(g)$ is an upper 
triangular matirx. If $\left|a_{v}\right| \rightarrow 0$, then $r_{\infty}^{\prime}=\lim _{v \rightarrow \infty} a_{v}^{2} r_{v}=0$. In this case, for any $g \in \Gamma, \eta(g)$ is an upper triangular matrix. But $\rho, \eta \in \bar{U} \subset R^{\prime}(\Gamma)$. This is a contradiction.

Proposition 2.6. The quotient space $R^{\prime}(\Gamma) / P G L_{2}(\mathbf{R})$ has the structure of a 6g-6 dimensional real analytic manifold such that the natural projection

$$
R^{\prime}(\Gamma) \rightarrow R^{\prime}(\Gamma) / P G L_{2}(\mathbf{R})
$$

is a real analytic principal $P G L_{2}(\mathbf{R})$-bundle.

Proof. Let $\rho \in R^{\prime}(\Gamma)$ be fixed. Let us define a real analytic mapping $G$ by

$$
\begin{aligned}
G: P G L_{2}(\mathbb{R}) & \rightarrow R^{\prime}(\Gamma) \subset S L_{2}(\mathbf{R})^{2 g} \\
P & \mapsto P^{-1} \rho P .
\end{aligned}
$$

We first show that for $P \in P G L_{2}(\mathbf{R})$ the differential $\left(d G_{*}\right)_{P}$ at $P$ has maximal rank. We may assume that $P \in S L_{2}(\mathbf{R})$ (For the case $\left(\begin{array}{cc}-1 & 0 \\ 0 & 1\end{array}\right) P \in S L_{2}(\mathbf{R})$ is essentially the same procedure). Then

$$
\begin{aligned}
(d G)_{P}: s l_{2}(\mathbf{R}) & \rightarrow s l_{2}(\mathbf{R})^{2 g} \\
X & \mapsto\left(P^{-1} \rho\left(\gamma_{j}\right) P X-X P^{-1} \rho\left(\gamma_{j}\right) P\right)_{1 \leq j \leq 2 g}
\end{aligned}
$$

where $\left\{\gamma_{j}\right\}$ is the system of generators of $\Gamma$. If $(d G)_{P}(X)=0$ for $X \in s l_{2}(\mathbf{R})$, then $P^{-1} \rho(g) P X=X P^{-1} \rho(g) P$ for all $g \in \Gamma . \quad \rho \in R^{\prime}(\Gamma)$ implies that $X=0$. Hence $(d G)_{P}$ is injective. Therefore the mapping $G$ is regular and there exists an open neighborhood $\Delta$ of the identity $I \in P G L_{2}(\mathbf{R})$ such that $G: \Delta \rightarrow G(\Delta)$ is a real analytic homeomorphism. Thus, there exist an open neighborhood $U$ of $\rho$ and a real analytic submanifold $V$ of $U$ so that $V$ and $G(\Delta)$ are transversal at $\rho$. Then the real analytic mapping defined by

$$
\begin{aligned}
& \Delta \times V \rightarrow R^{\prime}(\Gamma) \\
& (P, \eta) \mapsto P^{-1} \eta P
\end{aligned}
$$

is regular at $(I, \rho)$. We may assume that this mapping is a real analytic homeomorphism $\Delta \times V \simeq U$. To complete the proof, it is enough to show that this mapping extends to a real analytic homeomorphism from $P G L_{2}(\mathbb{R}) \times V$ 
into $R^{\prime}(\mathbb{\Gamma})$. For this purpose it is only necessary to check that after restricting $V$ if necessary, no two points of $V$ are in the same $P G L_{2}(\mathbb{R})$ orbit. Suppose that there are sequences $\left(\rho_{v}\right),\left(\eta_{v}\right) \subset V$ and $P_{v} \in P G L_{2}(\mathbb{R})$ such that $P_{v}^{-1} \rho_{v} P_{v}=\eta_{v}$ and $\lim _{v \rightarrow \infty} \rho_{v}=\lim _{v \rightarrow \infty} \eta_{v}=\rho$. Then Lemma 2.3 shows that by taking subsequence, we may assume that $P_{v}$ converges to some $P \in P G L_{2}(\mathbb{R})$. Since

$$
P^{-1} \rho P=\lim _{v \rightarrow \infty} P_{v}^{-1} \rho_{v} P_{v}=\rho
$$

$P$ is identity by Lemma 2.2. Hence $P_{v} \in \Delta$ for sufficiently large $v$. But

$$
P_{v}^{-1} \rho_{v} P_{v}=\eta_{v}=P^{-1} \eta_{v} P
$$

and $\Delta \times V \simeq U$. This is a contradiction.

\section{§2.3. The Space of Characters of $\Gamma$}

As we have seen in Subsection $2.1, R(\mathbb{\Gamma})$ has the structure of a real algebraic set. Let $\mathbb{R}[R(\mathbb{\Gamma})]$ be its affine coordinate ring i.e., the ring of polynomial functions on $R(\Gamma)$. Then the action of $P G L_{2}(\mathbb{R})$ on $R(\mathbb{T})$ induces the action of $P G L_{2}(\mathbb{R})$ on $\mathbb{R}[R(\Gamma)]$

$$
\begin{aligned}
P G L_{2}(\mathbb{R}) \times \mathbb{R}[R(\Gamma)] & \rightarrow \mathbb{R}[R(\mathbb{\Gamma})] \\
(\mathbb{P}, f(\rho)) & \mapsto f\left(\mathbb{P}^{-1} \rho P\right)
\end{aligned}
$$

and let $\mathbb{R}[R(\mathbb{\Gamma})]^{P G L_{2}(\mathbb{R})}$ be the ring of invariants of this action. For example the function $\tau_{h} \in \mathbb{R}[R(\Gamma)](h \in \Gamma)$ on $R(\Gamma)$ defined by

$$
\tau_{h}(\rho):=\operatorname{tr}(\rho(h))
$$

the trace of $\rho(h)$ for $\rho \in R(\Gamma)$ is an element of $\mathbb{R}[R(\Gamma)]^{P G L_{2}(\mathbb{R})}$. In fact $\mathbb{R}[R(\Gamma)]^{P G L_{2}(\mathbb{R})}$ is generated by the $\tau_{h}(h \in \Gamma)$ and is a finitely generated $\mathbb{R}$-subalgebra of $\mathbb{R}[R(\Gamma)]$ (see $[\mathrm{He}],[\mathrm{Ho}],[\mathrm{Pr}]$ ).

Let $X(\mathbb{\Gamma})$ be a real algebraic set whose affine coordinate ring $\mathbb{R}[X(\mathbb{\Gamma})]$ is isomorphic to $\mathbb{R}[R(\mathbb{\Gamma})]^{P G L_{2}(\mathbb{R})}$. And let $I_{h} \in \mathbb{R}[X(\mathbb{\Gamma})]$ correspond to $\tau_{h}$ $\in \mathbb{R}[R(\Gamma)]^{P G L_{2}(\mathbb{R})}$. Then $\mathbb{R}[X(\mathbb{\Gamma})]$ is generated by the $I_{h}(h \in \mathbb{\Gamma})$ as an $\mathbb{R}$-algebra. The injection

$$
\mathbb{R}[X(\Gamma)] \cong \mathbb{R}[R(\Gamma)]^{P G L_{2}(\mathbb{R})} \hookrightarrow \mathbb{R}[R(\Gamma)]
$$

induces a polynomial mapping 


$$
t: R(\Gamma) \rightarrow X(\Gamma)
$$

Because $\mathbb{R}[R(\Gamma)]^{P G L_{2}(\mathbb{R})}$ is generated by the $\tau_{h}(h \in \Gamma)$, for a represectation $\rho \in R(\Gamma), t(\rho)$ can be considered as the character $\chi_{\rho}$ of $\rho$

$$
\begin{aligned}
\chi_{\rho}: \Gamma & \rightarrow \mathbb{R} \\
h & \mapsto \operatorname{tr}(\rho(h))=\tau_{h}(\rho) .
\end{aligned}
$$

Therefore the image $t(R(\Gamma)) \subset X(\Gamma)$ of $R(\Gamma)$ under the mapping $t$ can be considered as the set of characters of $S L_{2}(\mathbb{R})$-representations of $\Gamma$. We call $X(\Gamma)$ the space of characters of $\Gamma$.

Moreover any element of $X(\Gamma)-t(R(\Gamma))$ can be considered as a character of an $S U(2)$-representation. To explain this we need to review briefly the theory of $S L_{2}(\mathrm{C})$-representations of $\Gamma$ following [C-S] and [M-S]. Let $R_{\mathrm{C}}(\Gamma)$ be the set of $S L_{2}(\mathbb{C})$-representations of $\Gamma$, then $R_{\mathbb{C}}(\Gamma)$ has the structure of a complex algebraic set and let $\mathbb{C}\left[R_{\mathbb{C}}(\Gamma)\right]$ be its affine coordinate ring. $P G L_{2}(\mathbb{C})$ acts on $R_{\mathbb{C}}(\Gamma)$ and also on $\mathbb{C}\left[R_{\mathbb{C}}(\Gamma)\right]$. Let $\mathbb{C}\left[R_{\mathbb{C}}(\Gamma)\right]^{P G L_{2}(\mathbb{C})}$ denote the ring of invariants of this action and let $X_{\mathbb{C}}(\Gamma)$ be a complex algebraic set whose affine coordinate ring $\mathbb{C}\left[X_{\mathbb{C}}(\Gamma)\right]$ is isomorphic to $\mathbb{C}\left[R_{\mathbb{C}}(\Gamma)\right]^{P G L_{2}(\mathbf{C})}$. Then the injection

$$
\mathbb{C}\left[X_{\mathbf{C}}(\Gamma)\right] \cong \mathbb{C}\left[R_{\mathbf{C}}(\Gamma)\right]^{P G L_{2}(\mathbf{C})} \hookrightarrow \mathbb{C}\left[R_{\mathbb{C}}(\Gamma)\right]
$$

induces the polynomial map

$$
t_{\mathbf{C}}: R_{\mathbf{C}}(\Gamma) \rightarrow X_{\mathbf{C}}(\Gamma)
$$

which is surjective. Since $R_{\mathbf{C}}(\Gamma), t_{\mathbf{C}}$ and $X_{\mathbf{C}}(\Gamma)$ are all defined over $\mathbf{Q}$, we can consider $X_{\mathbf{R}}(\Gamma)$, the set of real-valued points of $X_{\mathbf{C}}(\Gamma)$. Then we can consider $X_{\mathbb{R}}(\Gamma)$ as the set of real-valued characters of $S L_{2}(\mathbb{C})$-representations of $\Gamma$. It is known that any element of $X_{\mathbf{R}}(\Gamma)$ is either a character of $S L_{2}(\mathbb{R})$ or $S U(2)$-representation of $\Gamma$ ([M-S] Proposition 3.1.1).

If we consider the polynomial function $t r_{h} \in \mathbb{C}\left[R_{\mathbf{C}}(\Gamma)\right](h \in \Gamma)$ on $R_{\mathbf{C}}(\Gamma)$ defined by

$$
\operatorname{tr}_{h}(\rho):=\operatorname{tr}(\rho(h))
$$

for $\rho \in R_{\mathbf{C}}(\Gamma)$, then $t r_{h}$ is an element of $\mathbb{C}\left[R_{\mathbf{C}}(\Gamma)\right]^{P G L_{2}(\mathbf{C})}$, and we shall denote the corresponding element of $\mathbb{C}\left[X_{\mathbf{C}}(\Gamma)\right]$ also by $t r_{h}$ for the sake of simplicity. Then by regarding $R(\Gamma)$ as the set of real-valued points of $R_{\mathbf{C}}(\Gamma)$, we obtain a natural surjective homomorphism from $\mathbb{R}\left[X_{\mathbf{R}}(\Gamma)\right]$, the affine 
coordinate ring of $X_{\mathbf{R}}(\Gamma)$, to $\mathbf{R}[X(\Gamma)]$

$$
\begin{aligned}
\mathbf{R}\left[X_{\mathbf{R}}(\Gamma)\right] & \rightarrow \mathbf{R}[X(\Gamma)] \\
t r_{h} & \mapsto I_{h} .
\end{aligned}
$$

Therefore there is a canonical injection from $X(\Gamma)$ to $X_{\mathbf{R}}(\Gamma)$. Hence any element of $X(\Gamma)$ is either contained in $t(R(\Gamma))$ or can be considered as a character of a $S U(2)$-representation of $\Gamma$.

Next, we define the following subsets of $X(\Gamma)$

$$
\begin{aligned}
X^{\prime}(\Gamma) & :=t\left(R^{\prime}(\Gamma)\right) \\
U(\Gamma) & :=\left\{\chi \in X(\Gamma) \mid I_{[a, b]}(\chi) \neq 2 \text { for some } a, b \in \Gamma\right\} \\
& =X(\Gamma)-\bigcap_{a, b \in \Gamma}\left\{\chi \in X(\Gamma) \mid I_{[a, b]}(\chi)=2\right\} .
\end{aligned}
$$

Then $U(\Gamma)$ is open in $X(\Gamma)$. By Proposition $2.1 t^{-1}\left(X^{\prime}(\Gamma)\right)=R^{\prime}(\Gamma)$ and $X^{\prime}(\Gamma) \subset U(\Gamma)$.

Proposition 2.7. $X^{\prime}(\Gamma)$ is open in $U(\Gamma)$. Hence $X^{\prime}(\Gamma)$ is open in $X(\Gamma)$.

Proof. Let $V(\Gamma)$ be the set of characters of $S U(2)$-representations of $\Gamma$. Since $S U(2)$ is compact, $V(\Gamma)$ is compact in $X_{\mathbf{R}}(\Gamma)$. Hence $U(\Gamma)$ $=X^{\prime}(\Gamma) \cup(U(\Gamma) \cap V(\Gamma))$ and $(U(\Gamma) \cap V(\Gamma))$ is compact in $U(\Gamma)$. Therefore it is enough to show that $X^{\prime}(\Gamma) \cap(U(\Gamma) \cap V(\Gamma))=\phi$. For $\rho \in R^{\prime}(\Gamma)$, by Lemma 2.1 there exists $g \in \Gamma$ with $|\operatorname{tr}(\rho(g))|=\left|I_{g}(\rho)\right|>2$. On the other hand for any $S U(2)$-representation $\eta$ of $\Gamma$

$$
|\operatorname{tr}(\eta(h))|=\left|I_{h}(\eta)\right| \leq 2 \quad \text { for any } h \in \Gamma .
$$

Therefore $X^{\prime}(\Gamma) \cap(U(\Gamma) \cap V(\Gamma))=\phi$.

Next we will show that the restriction of the mapping $t$ to $R^{\prime}(\Gamma)$

$$
t: R^{\prime}(\Gamma) \rightarrow X^{\prime}(\Gamma)
$$

is a principal $P G L_{2}(\mathbf{R})$-bundle. By the result of Subsection 2.2, it is enough to show that $X^{\prime}(\Gamma)$ is the $P G L_{2}(\mathbf{R})$ adjoint quotient of $R^{\prime}(\Gamma)$. For this purpose, we need to prepare two lemmas which are $S L_{2}(\mathbf{R})$ versions of the results in 
[C-S] and [M-S].

Lemma 2.4. ([C-S, Proposition 1.5.2])

For $\rho_{1}, \rho_{2} \in R^{\prime}(\Gamma)$, we assume that $t\left(\rho_{1}\right)=t\left(\rho_{2}\right)$, in other words, they have the same character $\chi_{\rho_{1}}=\chi_{\rho_{2}}$. Then there is a $P \in P G L_{2}(\mathbf{R})$ such that $\rho_{2}=P^{-1} \rho_{1} P$.

Proof. By Lemma 2.1 and the assumption $\chi_{\rho_{1}}=\chi_{\rho_{2}}$, there exist $g \in \Gamma$ and $Q_{1}, Q_{2} \in P G L_{2}(\mathbf{R})$ such that

$$
Q_{1}^{-1} \rho_{1}(g) Q_{1}=Q_{2}^{-1} \rho_{2}(g) Q_{2}=\left(\begin{array}{cc}
\alpha & 0 \\
0 & \frac{1}{\alpha}
\end{array}\right) \quad(\alpha \neq \pm 1)
$$

Because $\rho_{1}$ is irreducible, there exist $h \in \Gamma$ and a diagonal matrix $R_{1} \in P G L_{2}(\mathbf{R})$ such that

$$
\left(Q_{1} R_{1}\right)^{-1} \rho_{1}(h)\left(Q_{1} R_{1}\right)=\left(\begin{array}{ll}
a_{1} & 1 \\
c_{1} & d_{1}
\end{array}\right) \quad\left(c_{1} \neq 0\right) .
$$

Moreover because $\chi_{\rho_{1}}([g, h])=\chi_{\rho_{2}}([g, h]) \neq 2$, there is a diagonal matrix $R_{2} \in P G L_{2}(\mathbf{R})$ such that

$$
\left(Q_{2} R_{2}\right)^{-1} \rho_{2}(h)\left(Q_{2} R_{2}\right)=\left(\begin{array}{ll}
a_{2} & 1 \\
c_{2} & d_{2}
\end{array}\right) \quad\left(c_{2} \neq 0\right) .
$$

For any $\gamma \in \Gamma$ put

$$
\begin{aligned}
& \left(Q_{1} R_{1}\right)^{-1} \rho_{1}(\gamma)\left(Q_{1} R_{1}\right)=\left(\begin{array}{ll}
p_{1} & q_{1} \\
r_{1} & s_{1}
\end{array}\right) \\
& \left(Q_{2} R_{2}\right)^{-1} \rho_{2}(\gamma)\left(Q_{2} R_{2}\right)=\left(\begin{array}{ll}
p_{2} & q_{2} \\
r_{2} & s_{2}
\end{array}\right) .
\end{aligned}
$$

Then $\chi_{\rho_{1}}(\gamma)=\chi_{\rho_{2}}(\gamma)$ and $\chi_{\rho_{1}}(g \gamma)=\chi_{\rho_{2}}(g \gamma)$ implies that $p_{1}=p_{2}$ and $s_{1}=s_{2}$. If we put $\gamma=h$, then we obtain that $a_{1}=a_{2}$ and $d_{1}=d_{2} . \quad$ Then $\operatorname{det}\left(\rho_{1}(h)\right)=\operatorname{det}\left(\rho_{2}(h)\right)=1$ shows $c_{1}=c_{2}$. If we take $h \gamma$ for our $\gamma$, then we obtain $r_{1}=r_{2}$ and $q_{1}=q_{2}$. Therefore if we take $P=\left(Q_{1} R_{1}\right)\left(Q_{2} R_{2}\right)^{-1}$, then $\rho_{2}=P^{-1} \rho_{1} P$.

Lemma 2.5. ([M-S, Lemma 3.1.7])

For a subset $U$ of $X^{\prime}(\Gamma)$ we assume that $t^{-1}(U)$ is open in $R^{\prime}(\Gamma)$, hence open in $[R(\Gamma)]$. Then $U$ is open in $X^{\prime}(\Gamma)$ hence in $X(\Gamma)$. 
Proof. Suppose that there exists a sequence $\left\{\chi_{i}\right\} \subset X^{\prime}(\mathbb{\Gamma})-U$ so that $\chi_{i}$ converges to $\chi \in U$. Let $\rho \in R^{\prime}(\Gamma)$ satisfy $\chi_{\rho}=\chi$. By Lemma 2.1 we may assume that there exist $g, h \in \mathbb{\Gamma}$ such that

$$
\begin{gathered}
\rho(g)=\left(\begin{array}{ll}
\lambda & 0 \\
0 & \frac{1}{\lambda}
\end{array}\right) \quad(\lambda \neq \pm 1) \\
\rho(h)=\left(\begin{array}{ll}
a & 1 \\
c & d
\end{array}\right) \quad(c \neq 0) .
\end{gathered}
$$

Since $I_{[g . h]}(\chi) \neq 2$, by taking a subsequence of $\left\{\chi_{i}\right\}$ we may suppose that

$$
\left|I_{g}\left(\chi_{i}\right)\right|>2 \text { and } I_{[g . h]}\left(\chi_{i}\right) \neq 2 .
$$

Let $\rho_{i} \in R^{\prime}(\Gamma)-t^{-1}(U)$ satisfy $\chi_{\rho_{i}}=\chi_{i}$. Then since $\left|I_{g}\left(\chi_{i}\right)\right|>2$ we may suppose that

$$
\rho_{i}(g)=\left(\begin{array}{cc}
\lambda_{i} & 0 \\
0 & \frac{1}{\lambda_{i}}
\end{array}\right) \quad\left(\lambda_{i} \neq \pm 1\right) .
$$

Moreover as $I_{g}\left(\chi_{i}\right) \rightarrow I_{g}(\chi)$ we may assume that $\lambda_{i} \rightarrow \lambda$.

For $\gamma \in \Gamma$ put

$$
\rho_{i}(\gamma)=\left(\begin{array}{cc}
x_{i} & y_{i} \\
z_{i} & w_{i}
\end{array}\right), \quad \rho(\gamma)=\left(\begin{array}{ll}
x & y \\
z & w
\end{array}\right) .
$$

Then $I_{\gamma g}\left(\chi_{i}\right) \rightarrow I_{\gamma g}(\chi)$ and $I_{\gamma g^{2}}\left(\chi_{i}\right) \rightarrow I_{\gamma g^{2}}(\chi)$ shows that $x_{i} \rightarrow x$ and $w_{i} \rightarrow w$. On the other hand, since $\mathbb{I}_{[g . h]}\left(\chi_{i}\right) \neq 2$, we may suppose that

$$
\rho_{i}(h)=\left(\begin{array}{ll}
a_{i} & 1 \\
c_{i} & d_{i}
\end{array}\right) \quad\left(c_{i} \neq 0\right) .
$$

Then replacing $\gamma$ by $h$, we have $a_{i} \rightarrow a, d_{i} \rightarrow d$ and $c_{i} \rightarrow c$. Moreover replacing $\gamma$ by $h \gamma$, we have $y_{i} \rightarrow y$ and $z_{i} \rightarrow z$. Hence $\rho_{i}$ converges to $\rho$. But $\rho_{i} \in R^{\prime}(\Gamma)-t^{-1}(U)$ and $\rho \in t^{-1}(U)$. This is a contradiction.

By Lemmas 2.4 and 2.5, we conclude that

Proposition 2.8. $\quad t: R^{\prime}(\Gamma) \rightarrow X^{\prime}(\Gamma)$ can be considered as the quotient map of $R^{\prime}(\Gamma)$ under the action of $P G L_{2}(\mathbb{R})$, i.e., 


$$
X^{\prime}(\Gamma)=R^{\prime}(\Gamma) / P G L_{2}(\mathbb{R})
$$

Therefore by the result of Subsection 2.2, $t: R^{\prime}(\Gamma) \rightarrow X^{\prime}(\Gamma)$ is a principal $P G L_{2}(\mathbf{R})$-bundle.

Define the closed subset $X_{0}(\Gamma)$ of $X(\Gamma)$ by

$$
\begin{array}{r}
X_{0}(\Gamma):=\left\{\chi \in X(\Gamma)\left|I_{[g . h]}(\chi)-2\right|+\left|I_{h}(\chi)^{2}-4\right| \geq 1\right. \\
\text { for } g, h \in \Gamma \text { with } g h \neq h g\} .
\end{array}
$$

Then the proof of Proposition 2.4 implies that $t\left(R_{0}(\Gamma)\right) \subset X_{0}(\Gamma)$.

Proposition 2.9. 1. $X_{0}(\Gamma)=t\left(R_{0}(\Gamma)\right)$.

2. $X_{0}(\Gamma)$ is open in $X^{\prime}(\Gamma)$ hence open in $X(\Gamma)$.

3. $t^{-1}\left(X_{0}(\Gamma)\right)=R_{0}(\Gamma)$.

Proof. 1. Any representation of $\Gamma$ to $S L_{2}(\mathbf{C})$ is discrete and faithful if and only if it satisfies the inequalities of Jørgensen which we saw in the proof of Proposition 2.4. But there are no discrete and faithful $S U(2)$-representations of $\Gamma$ because $S U(2)$ is compact and $\Gamma$ is an infinite group. Hence $X_{0}(\Gamma) \subset t(R(\Gamma))$ and it follows that $X_{0}(\Gamma)=t\left(R_{0}(\Gamma)\right)$.

2. $R_{0}(\Gamma) \subset R^{\prime}(\Gamma)$ implies $X_{0}(\Gamma) \subset X^{\prime}(\Gamma)$. Because $R_{0}(\Gamma)$ is open in $R(\Gamma)$ and $t: R^{\prime}(\Gamma) \rightarrow X^{\prime}(\Gamma)$ is an open map by Proposition 2.6, $X_{0}(\Gamma)$ is open in $X^{\prime}(\Gamma)$.

3. This is immediate from the proof of Proposition 2.4.

Corollary 2.4. $\quad X_{0}(\Gamma)$ is open and closed in $X(\Gamma)$. Therefore $X_{0}(\Gamma)$ consists of finitely many connected components of $X(\Gamma)$. Hence it is a semialgebraic subset of $X(\Gamma)$.

Corollary 2.5. $t: R_{0}(\Gamma) \rightarrow X_{0}(\Gamma)$ is also a principal $P G L_{2}(\mathbb{R})$-bundle. Hence $X_{0}(\Gamma)$ can be considered as the $P G L_{2}(\mathbb{R})$ adjoint quotient of $R_{0}(\Gamma)$, i.e., $X_{0}(\Gamma)=R_{0}(\Gamma) / P G L_{2}(\mathbf{R})$.

We summarize the results of this subsection as the following diagram 


$$
\begin{array}{lcccc}
R(\Gamma) & \supset & R^{\prime}(\Gamma) & \supset & R_{0}(\Gamma) \\
t \downarrow & \downarrow & \downarrow & P G L_{2}(\mathbf{R}) \text {-bundle } \\
X(\Gamma) & \supset & X^{\prime}(\Gamma) & \supset & X_{0}(\Gamma)=R_{0}(\Gamma) / P G L_{2}(\mathbf{R}) .
\end{array}
$$

\section{§2.4. The Relation Between $S L_{2}(\mathbf{R})$ - and $P S L_{2}(\mathbf{R})$-Representations of $\Gamma$}

Next we consider the relation between $S L_{2}(\mathbf{R})$ - and $P S L_{2}(\mathbf{R})$-representations of the surface group $\Gamma$.

The group $\operatorname{Hom}(\Gamma, \mathbb{Z} / 2 \mathbb{Z})\left(\cong(\mathbb{Z} / 2 \mathbb{Z})^{2 g}\right)$ acts on $R(\Gamma)$ as follows. For any $\mu \in \operatorname{Hom}(\Gamma, \mathbb{Z} / 2 \mathbb{Z})$ and $\rho \in R(\Gamma)$, we define the representation $\mu \cdot \rho R(\Gamma)$ by

$$
\mu \cdot \rho(h):=\mu(h) \cdot \rho(h) \quad(\text { for all } h \in \Gamma)
$$

where $\mathbb{Z} / 2 \mathbb{Z}$ acts on $S L_{2}(\mathbf{R})$ by multiplication by \pm 1 .

Proposition 2.10. ([Pa], [S-S ], [O2])

Let $\xi: \Gamma \rightarrow P S L_{2}(\mathbf{R})$ be a discrete and faithful $P S L_{2}(\mathbf{R})$ representation. Let $A_{i}, B_{i} \in S L_{2}(\mathbf{R})$ denote any representatives of $\xi\left(\alpha_{i}\right), \xi\left(\beta_{i}\right) \in P S L_{2}(\mathbf{R})(i=1, \cdots, g)$. Then

$$
\prod_{i=1}^{g}\left[A_{i}, B_{i}\right]=\left(\begin{array}{ll}
1 & 0 \\
0 & 1
\end{array}\right) .
$$

In other words, $\xi$ can always be lifted to a representation $\rho \in R_{0}(\Gamma)$ and the set of all liftings of $\xi$ is equal to the $\operatorname{Hom}(\Gamma, \mathbb{Z} / 2 \mathbb{Z})$ orbit of $\rho$ in $R_{0}(\Gamma)$.

$$
\begin{array}{rr} 
& S L_{2}(\mathbb{R}) \\
\rho_{\nearrow} & \downarrow \text { proj. } \\
\Gamma \stackrel{\xi}{\rightarrow} & P S L_{2}(\mathbb{R})
\end{array}
$$

Proof. We briefly review what Seppälä and Sorvali showed in their paper [S-S].

Let $\xi$ be a discrete and faithful $P S L_{2}(\mathbf{R})$ representation. Suppose $A_{i}, B_{i} \in S L_{2}(\mathbb{R})(i=1, \cdots, g)$ denote any representatives of $\xi\left(\alpha_{i}\right), \xi\left(\beta_{i}\right) \in P S L_{2}(\mathbb{R})$. Then they showed that 


$$
\begin{gathered}
\operatorname{tr}\left(\left[A_{i}, B_{i}\right]\right)<-2(i=1, \cdots, g) \\
\operatorname{tr}\left(\left[A_{1}, B_{1}\right] \cdots\left[A_{j}, B_{j}\right]\right)<-2(j=2, \cdots, g-1) .
\end{gathered}
$$

In particular

$$
\begin{array}{r}
\operatorname{tr}\left(\left[A_{g}, B_{g}\right]\right)<-2 \\
\operatorname{tr}\left(\left[A_{1}, B_{1}\right] \cdots\left[A_{g-1}, B_{g-1}\right]\right)<-2 .
\end{array}
$$

We may suppose that $\left[A_{1}, B_{1}\right] \cdots\left[A_{g-1}, B_{g-1}\right]$ is a diagonal matrix. Then $\left[A_{g}, B_{g}\right]$ must be also diagonal, hence the above inequalities imply the conclusion.

Corollary 2.6. 1. $\operatorname{Hom}(\Gamma, \mathbf{Z} / 2 \mathrm{Z})$ acts on $R_{0}(\Gamma)$ and the quotient space $\operatorname{Hom}(\Gamma, \mathbf{Z} / 2 \mathbf{Z}) \backslash R_{0}(\Gamma)$ can be considered as the set of discrete and faithful $\mathrm{PSL}_{2}(\mathbf{R})$-representations of $\Gamma$.

2. Through the mapping $t, \operatorname{Hom}(\Gamma, \mathbf{Z} / 2 \mathbf{Z})$ also acts on $X_{0}(\Gamma)$ and the quotient space $\operatorname{Hom}(\Gamma, \mathbf{Z} / 2 \mathrm{Z}) \backslash X_{0}(\Gamma)$ can be considered as the $P G L_{2}(\mathbf{R})$-adjoint quotient of the set of discrete and faithful $P S L_{2}(\mathbf{R})$-representations of $\Gamma$.

We call this set the Teichmüller space $T_{g}$

$$
\begin{aligned}
T_{g} & :=\operatorname{Hom}(\Gamma, \mathbf{Z} / 2 \mathbf{Z}) \backslash X_{0}(\Gamma) \\
& =\operatorname{Hom}(\Gamma, \mathbf{Z} / 2 \mathbf{Z}) \backslash R_{0}(\Gamma) / P G L_{2}(\mathbf{R}) .
\end{aligned}
$$

Remark. ([Sa 1]) For any $\rho \in R_{0}(\Gamma)$, let $\pi$ be the projection

$$
\pi: \mathbf{H} \rightarrow M:=\rho(\Gamma) \backslash \mathbf{H}
$$

and put $p:=\pi(\sqrt{-1}) \in M$. Then the isomorphism from $\rho(\Gamma)$ to the fundamental group $\pi_{1}(M, p)$ of $M$ with a base point $p$

$$
\imath: \rho(\Gamma) \simeq \pi_{1}(M, p)
$$

is uniquely determined. Put $\left.\left.a_{i}:=l\left(\rho\left(\alpha_{i}\right)\right)\right), b_{i}:=l\left(\rho\left(\beta_{i}\right)\right)\right) \in \pi_{1}(M, \rho) \quad(i=1, \cdots, g)$. We say a representation $\rho$ is orientation preserving (resp. reversing), if, with respect to the orientation on $M$ coming from the complex structure on $\mathbf{H}$,

$$
\left\langle\left[a_{i}\right],\left[b_{i}\right]\right\rangle=\delta_{i j}\left(\text { resp. }-\delta_{i j}\right),\left\langle\left[a_{i}\right],\left[a_{j}\right]\right\rangle=\left\langle\left[b_{i}\right],\left[b_{j}\right]\right\rangle=0
$$


$(i, j=1, \cdots, g)$ where $\left[a_{i}\right]$ is the homology class of $a_{i}$ and $<,>$ is the intersection pairing on $H_{1}(M, \mathbb{Z})$. If $R_{0}^{+}(\Gamma)$ (resp. $R_{0}^{-}(\Gamma)$ ) denotes the set of orientation preserving (resp. reversing), discrete and faithful $S L_{2}(\mathbb{R})$-representations of $\Gamma$, then $R_{0}(\mathbb{\Gamma})$ is the disjoint union of $R_{0}^{+}(\mathbb{\Gamma})$ and $R_{0}^{-}(\Gamma)$ and

$$
\begin{aligned}
X_{0}(\Gamma) & =R_{0}(\Gamma) / P G L_{2}(\mathbb{R})=R_{0}^{+}(\Gamma) / P S L_{2}(\mathbb{R}) . \\
T_{g} & =H o m(\Gamma, \mathbb{Z} / 2 \mathbb{Z}) \backslash X_{0}(\Gamma) \\
& =\operatorname{Hom}(\Gamma, \mathbb{Z} / 2 \mathbb{Z}) \backslash R_{0}^{+}(\Gamma) / P S L_{2}(\mathbb{R}) .
\end{aligned}
$$

Proposition 2.3 implies $\left|I_{h}\right|>2$ (for all $h(\neq$ identity) $\in \mathbb{\Gamma})$ on $X_{0}(\mathbb{\Gamma})$. Hence the sign of $I_{h}$ is constant on each connected component of $X_{0}(\Gamma)$. This means that $\operatorname{Hom}(\Gamma, \mathbb{Z} / 2 \mathbb{Z})$ permutes the set of connected components of $X_{0}(\mathbb{\Gamma})$ freely. Thus

Corollary 2.7. The quotient map $X_{0}(\Gamma) \rightarrow T_{g}$ is an unramified $(\mathbb{Z} / 2 \mathbb{Z})^{2 g_{-}}$ covering. Hence by taking any lifting of this mapping, we can consider $T_{g}$ as a finite union of connected components of $X_{0}(\Gamma)$. Therefore $T_{g}$ can be considered as a semialgebraic subset of $X_{0}(\Gamma)$.

Corollary 2.8. If $\pi_{0}\left(X_{0}(\Gamma)\right)$ denotes the number of connected components of $X_{0}(\Gamma)$, the order of $\operatorname{Hom}(\Gamma, \mathbb{Z} / 2 \mathbb{Z})$ divides $\pi_{0}\left(X_{0}(\Gamma)\right)$. In particular

$$
2^{2 g} \leq \pi_{0}\left(X_{0}(\Gamma)\right)
$$

We summarize the result of this subsection as the following diagram.

$$
\begin{aligned}
\operatorname{Hom}\left(\Gamma, S L_{2}(\mathbb{R})\right)= & R(\Gamma) \supset R_{0}(\Gamma) \\
{ }^{t} \downarrow & \downarrow \\
X(\Gamma) \supset X_{0}(\Gamma) & =\quad R_{0}(\Gamma) / P G L_{2}(\mathbb{R}) \\
\downarrow & \\
& T_{g}=\operatorname{Hom}(\Gamma, \mathbb{Z} / 2 \mathbb{Z}) \backslash X_{0}(\Gamma)
\end{aligned}
$$

§3. Semialgebraic Description of Teichmüller Space $T_{g}(g=2)$

In this section, by construction global coordinates on $X_{0}(\Gamma)$, we will give a semialgebraic description of the Teichmüller space $T_{2}$, which we will use to 
show its connectivity and contractibility. For this purpose, we need to find some semialgebraic subset $S(\Gamma)$ of $X(\Gamma)$ containing $X_{0}(\Gamma)$ whose presentation as a semialgebraic set and topological structure are both simple.

\section{§3.1. Definition of the Semialgebraic Subset $S(\Gamma)$ of $X(\Gamma)$}

We define an open semialgebraic subset $S(\Gamma)$ of $X(\Gamma)$ by

$$
S(\Gamma):=\left\{\chi \in X(\Gamma) \mid I_{c_{1}}(\chi)<-2\right\}
$$

where $c_{1}:=\left[\alpha_{1}, \beta_{1}\right]=\left[\alpha_{2}, \beta_{2}\right]^{-1} \in \Gamma$.

Proposition 3.1. $S(\Gamma) \subset X^{\prime}(\Gamma)$. Hence $t^{-1}(S(\Gamma)) \stackrel{t}{\rightarrow} S(\Gamma)$ is a $P G L_{2}(\mathbb{R})$ bundle, and $S(\Gamma)$ can be considered as the $P G L_{2}(\mathbb{R})$-adjoint quotient of $t^{-1}(S(\Gamma))$, i.e.,

$$
S(\Gamma)=t^{-1}(S(\Gamma)) / P G L_{2}(\mathbf{R})
$$

Proof. First we show

$$
S(\Gamma) \cap(X(\Gamma)-t(R(\Gamma)))=\phi
$$

As we have seen in Subsection 2.3, any element of $X(\Gamma)-t(R(\Gamma))$ can be considered as a character of an $\mathrm{SU}(2)$-representation of $\Gamma$. Thus for $\chi \in X(\Gamma)-t(R(\Gamma))$

$$
\left|I_{h}(\chi)\right| \leq 2 \quad \text { for } h \in \Gamma .
$$

This means that $S(\Gamma) \subset t(R(\Gamma))$. On the other hand, Proposition 2.1 shows that $S(\Gamma) \subset X^{\prime}(\Gamma)$.

The next result is due to Keen, Okumura and Seppälä-Sorvali ([K1], [K2], [K3], [O1], [O2], [S-S ]).

Proposition 3.2. $X_{0}(\Gamma) \subset S(\Gamma)$.

Proof. Any element $\rho=\left(A_{1}, B_{1}, A_{2}, B_{2}\right)$ of $R_{0}(\Gamma)$ induces a discrete and faithful $\operatorname{PSL}_{2}(\mathbf{R})$-representation of $\Gamma$. Hence we have seen in the proof of Proposition 2.10 that

$$
\operatorname{tr}\left(\left[A_{1}, B_{1}\right]\right)<-2
$$


This completes the proof.

The above arguments give rise to the following diagram.

\section{Corollary 3.1.}

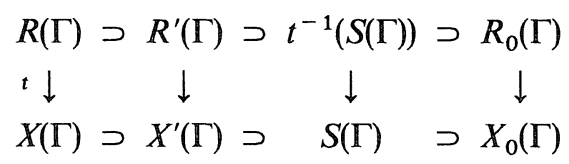

\section{§3.2. Topological Structure of $S(\Gamma)$}

In this subsection, by constructing global coordinates on $S(\Gamma)$, we will show that $S(\Gamma)$ consists of $2^{4} \times 2$ connected components such that each component is a 6 dimensional cell. For this purpose we need some preliminaries.

Let us define a polynomial mapping $f$ from $X(\Gamma)$ to $\mathbb{R}^{6}$ : For any $\chi \in X(\Gamma)$, let

$$
f(\chi):=\left(I_{\alpha_{1}}(\chi), I_{\beta_{1}}(\chi), I_{\alpha_{1} \beta_{1}}(\chi), I_{\alpha_{2}}(\chi), I_{\beta_{2}}(\chi), I_{\alpha_{2} \beta_{2}}(\chi)\right)
$$

By the definition of $I_{h}(h \in \Gamma)$, for any $\rho \in R(\Gamma)$

$$
\begin{gathered}
f \circ t(\rho)=\left(\operatorname{tr}\left(\rho\left(\alpha_{1}\right)\right), \operatorname{tr}\left(\rho\left(\beta_{1}\right)\right), \cdots, \operatorname{tr}\left(\rho\left(\alpha_{2} \beta_{2}\right)\right)\right) . \\
R(\Gamma) \\
t \downarrow \stackrel{f \circ t}{\searrow} \\
X(\Gamma) \stackrel{f}{\rightarrow} \mathbb{R}^{6}
\end{gathered}
$$

We denote the coordinates $\left(x_{1}, x_{2}, x_{3}, y_{1}, y_{2}, y_{3}\right)$ on $\mathbf{R}^{6}$ by $(\vec{x}, \vec{y})$ for the sake of simplicity. Next we define a polynomial function $\kappa(x, y, z)$ on $\mathbf{R}^{3}$ by

$$
\kappa(x, y, z):=x^{2}+y^{2}+z^{2}-x y z-2 .
$$

Easy calculation shows the following lemma ([F], $[\mathrm{Go}])$.

Lemma 3.1. 1. For any $A, B \in S L_{2}(\mathbf{R})$

$$
\kappa(\operatorname{tr}(A), \operatorname{tr}(B), \operatorname{tr}(A B))=\operatorname{tr}([A, B]) .
$$


2. If $(x, y, z) \in \mathbf{R}^{3}$ satisfies $\kappa(x, y, z)<-2$, then

$$
|x|>2, \quad|y|>2, \quad|z|>2 \text { and } x \cdot y \cdot z>0 .
$$

In particular if we put

$$
V_{-}=\left\{(\vec{x}, \vec{y}) \in \mathbf{R}^{6} \mid \kappa(\vec{x})=\kappa(\vec{y})<-2\right\}
$$

then from the definition of $S(\Gamma)$, we have $f(S(\Gamma)) \subset V_{-}$. In fact we will see in Proposition 3.3 that $f(S(\Gamma))=V_{-}$.

Lemma 3.2. $\quad V_{-} \subset \mathbf{R}^{6}$ consists of $2^{4}$ connected components such that each components is a 5-dimensional cell. More precisely, put $U:=V_{-} \cap\left\{(\vec{x}, \vec{y}) \in \mathbf{R}^{6}\right.$ $\left.\mid x_{i}>0, y_{i}>0(i=1,2)\right\}$ and define the action of $(\mathbf{Z} / 2 \mathbf{Z})^{4}$ on $\mathbf{R}^{6}$ by multiplication by \pm 1 on the $x_{i}$ and $y_{i}(i=1,2)$. Then $U$ is a 5 dimensional cell and $V_{-}$can be written as

$$
V_{-}=\coprod_{\gamma \in(\mathbf{Z} / 2 \mathbf{Z})^{4}} \gamma(U) \quad \text { (disjoint union). }
$$

Proof. For $r<-2$ put

$$
W_{r}:=\left\{(x, y, z) \in \mathbf{R}^{3} \mid \kappa(x, y, z)=r, x>0, y>0, z>0\right\}
$$

and $u:=x-y, v:=x+y$ for $(x, y, z) \in W_{r}$. Then by Lemma 3.1.2

$$
v=\sqrt{\frac{z+2}{z-2} u^{2}-\frac{4}{z-2}\left(2+r-z^{2}\right)}>0 .
$$

Hence the next mapping is a homeomorphism, and consequently $W_{r}$ is a 2 demensional cell.

$$
\begin{aligned}
W_{\mathbf{r}} & \simeq \mathbf{R} \times\{z \in \mathbf{R} \mid z>2\} . \\
(x, y, z) & \mapsto(u, z)
\end{aligned}
$$

Since $U$ is a fiber bundle over the base space $\{r \in \mathbf{R} \mid r<-2\}$ with fibers $W_{r} \times W_{r}$, $U$ is a 5 dimensional cell and by Lemma 3.1 .2 


$$
V_{-}=\coprod_{\gamma \in(\mathbf{Z} / 2 \mathbf{Z})^{4}} \gamma(U)
$$

The next lemma can be shown directly by calculation, but it is a key lemma in the whole story of this section.

Lemma 3.3. Let $(A, B) \in S L_{2}(\mathbb{R})^{2}$ be a pair of hyperbolic matrices which satisfies the following condition

$$
\left.[A, B]=\left(\begin{array}{cc}
\lambda & 0 \\
0 & \frac{1}{\lambda}
\end{array}\right) \quad(\lambda<-1) . \cdots 1\right)
$$

Put $(x, y, z):=(\operatorname{tr}(A), \operatorname{tr}(B), \operatorname{tr}(A B)) . \quad$ Then $\kappa(x, y, z)<-2$, and there exists a constant $k \in \mathbb{R}^{*}:=\mathbb{R}-\{0\}$ such that $A$ and $B$ can be written as

$$
A=\left(\begin{array}{cc}
\frac{1}{\lambda+1} x & \frac{1}{k}\left\{\frac{\lambda}{(\lambda+1)^{2}} x^{2}-1\right\} \\
k & \frac{1}{\lambda+1} x
\end{array}\right)
$$

$$
B=\left|\begin{array}{cc}
\frac{1}{\lambda+1} y & \frac{1}{k}\left\{\frac{1}{\lambda+1} z-\frac{\lambda}{(\lambda+1)^{2}} x y\right\} \\
\frac{\lambda}{(\lambda+1)^{2}} y^{2}-1 & \frac{\lambda}{\lambda+1} y \\
\frac{1}{\lambda+1} z-\frac{\lambda}{(\lambda+1)^{2}} x y &
\end{array}\right|
$$

Conversely for any $k \in \mathbb{R}^{*}$ and $(x, y, z) \in \mathbb{R}^{3}$ with $\kappa(x, y, z)<-2$, define $\lambda<-1$ by $\lambda+\frac{1}{\lambda}=\kappa(x, y, z)$. Then the pair of matrices $(A, B) \in S L_{2}(\mathbf{R})^{2}$ defined by condition 2) satisfies 1) and $(x, y, z)=(\operatorname{tr}(A), \operatorname{tr}(B), \operatorname{tr}(A B))$.

Since the pair $(A, B) \in S L_{2}(\mathbb{R})^{2}$ defined by the above condition 2$)$ is uniquely determined by $k \in \mathbb{R}^{*}$ and $(x, y, z) \in \mathbb{R}^{3}$ with $\kappa(x, y, z)<-2$, we write it as 


$$
(A, B)=(A(x, y, z, k), B(x, y, z, k))
$$

Now we can show the main result of this subsection.

Proposition 3.3. $S(\Gamma)$ consists of $2^{4} \times 2$ connected components such that each component is a 6-dimensional cell.

Proof. First, we define the mapping $\Psi$

$$
\Psi: t^{-1}(S(\Gamma)) \rightarrow \mathbb{R}^{*} \times V_{-} \times P G L_{2}(\mathbb{R})
$$

as follows: For any $\rho=\left(A_{1}, B_{1}, A_{2}, B_{2}\right) \in t^{-1}(S(\Gamma))$, we first diagonalize $\left[A_{1}, B_{1}\right]$. More precisely, by using Lemma 3.3 , we can choose $P \in P G L_{2}(\mathbf{R})$ uniquely such that by use of the notations of Lemma 3.3, $\left(P A_{i} P^{-1}, P B_{i} P^{-1}\right)$ $(i=1,2)$ can be written as

$$
\begin{aligned}
& P A_{1} P^{-1}=A\left(\operatorname{tr}\left(A_{1}\right), \operatorname{tr}\left(B_{1}\right), \operatorname{tr}\left(A_{1} B_{1}\right), 1\right) \\
& P B_{1} P^{-1}=B\left(\operatorname{tr}\left(A_{1}\right), \operatorname{tr}\left(B_{1}\right), \operatorname{tr}\left(A_{1} B_{1}\right), 1\right) \\
& P A_{2} P^{-1}=\left(\begin{array}{cc}
0 & 1 \\
-1 & 0
\end{array}\right) A\left(\operatorname{tr}\left(A_{2}\right), \operatorname{tr}\left(B_{2}\right), \operatorname{tr}\left(A_{2} B_{2}\right), k\right)\left(\begin{array}{cc}
0 & -1 \\
1 & 0
\end{array}\right) \\
& P B_{2} P^{-1}=\left(\begin{array}{cc}
0 & 1 \\
-1 & 0
\end{array}\right) B\left(\operatorname{tr}\left(A_{2}\right), \operatorname{tr}\left(B_{2}\right), \operatorname{tr}\left(A_{2} B_{2}\right), k\right)\left(\begin{array}{cc}
0 & -1 \\
1 & 0
\end{array}\right)
\end{aligned}
$$

where $k \in \mathbb{R}^{*}$ is some constant. We define the mapping $\Psi$ by

$$
\begin{aligned}
\Psi: t^{-1}(S(\Gamma)) & \rightarrow \mathbf{R}^{*} \times V_{-} \times P G L_{2}(\mathbf{R}) \\
\rho & \mapsto(k, f \circ t(\rho), P) .
\end{aligned}
$$

Lemma 3.3 implies that $\Psi$ is bijective and, in fact, a homeomorphism. From the difinition, $\Psi$ is $P G L_{2}(\mathbf{R})$-equivariant. Hence it induces a homeomorphism $\Phi$ from $S(\Gamma)$ to $\mathbb{R}^{*} \times V_{-}$as follows.

$$
\begin{array}{ccc}
t^{-1}(S(\Gamma)) & \stackrel{\Psi}{\simeq} \mathbf{R}^{*} \times V_{-} \times P G L_{2}(\mathbf{R}) \\
t \downarrow & & \downarrow \\
S(\Gamma) & \stackrel{\Phi}{\simeq} & \mathbf{R}^{*} \times V_{-} .
\end{array}
$$


Moreover by Lemma $3.2, \mathbf{R}^{*} \times V_{\text {- }}$ consists of $2^{4} \times 2$ connected components such that each component is a 6-dimensional cell.

\section{§3.3. Cell Structure of Teichmüller Space $T_{2}$}

Next we consider conditions which characterize the connected components of $X_{0}(\Gamma)$ in $S(\Gamma)$. By the definition of $\Phi$ in the proof of Proposition 3.3, the first component $k$ of $\Phi$ can be considered as a function on $S(\Gamma)$.

Proposition 3.4. Suppose that $U \subseteq S(\Gamma)$ is a connected component on which the function $I_{\alpha_{1}} \cdot I_{\alpha_{2}} \cdot k$ is negative. Then there exists $\chi \in U$ such that $\chi$ is not contained in $X_{0}(\Gamma)$.

Proof. First we remark that on a connected component $U$ of $S(\Gamma)$, the signs of the functions $I_{\alpha_{1}} \cdot I_{\alpha_{2}}$, and $k$ are constant. We consider $(\vec{x}, \vec{y}) \in V_{-}$ satisfying $\left|x_{i}\right|=\left|y_{i}\right|=4 \quad(i=1,2,3)$. Then there are $2^{4}$ points of $V_{\text {- satisfying }}$ this condition. Since $f(U)$ is a connected component of $V_{-}$, take $\rho=$ $\left(A_{1}, B_{1}, A_{2}, B_{2}\right) \in t^{-1}(S(\Gamma))$ with $t(\rho) \in U$ and $f \circ t(\rho)=(\vec{x}, \vec{y})$. If $I_{\alpha_{1}}(t(\rho)) \cdot I_{\alpha_{2}}(t(\rho))$ $=\operatorname{tr}\left(A_{1}\right) \cdot \operatorname{tr}\left(A_{2}\right)=16>0$, then by using the presentation of $\rho=\left(A_{1}, B_{1}\right.$, $\left.A_{2}, B_{2}\right)$ in the proof of Proposition 3.3, $\operatorname{tr}\left(A_{1} A_{2}\right)=-2-k-\frac{4}{k}$ where we denote $k(\rho)$ by $k$ for the sake of simplicity. Hence if $k(\rho)=k=-2$ (i.e., $I_{\alpha_{1}} \cdot I_{\alpha_{2}} \cdot k<0$ on $U$ ), then $\operatorname{tr}\left(A_{1} A_{2}\right)=2$ and this means that $A_{1} A_{2} \in S L_{2}(\mathbf{R})$ is a parabolic matrix, thus $t(\rho)$ is not contained in $X_{0}(\Gamma)$. Similar argument holds for the case $I_{\alpha_{1}}(\rho) \cdot I_{\alpha_{2}}(\rho)=\operatorname{tr}\left(A_{1}\right) \cdot \operatorname{tr}\left(A_{2}\right)=-16<0$.

Since $X_{0}(\Gamma)$ consists of finitely many connected components of $X(\Gamma)$ by Corollary 2.4, Proposition 3.4 implies that there are 16 connected components of $S(\Gamma)$ on which the function $I_{\alpha_{1}} \cdot I_{\alpha_{2}} \cdot k$ is negative. Hence the number of connected components of $X_{0}(\Gamma), \pi_{0}\left(X_{0}(\Gamma)\right)$ is less than or equal to 16 . On the other hand, as the argument in Subsection 2.4 implies $\pi_{0}\left(X_{0}(\Gamma)\right) \geq 16$, we get following result.

Theorem 3.1. $\pi_{0}\left(X_{0}(\Gamma)\right)=16$. Thus

$$
T_{2}=\operatorname{Hom}(\Gamma, \mathbb{Z} / 2 \mathbb{Z}) \backslash X_{0}(\Gamma)
$$

is a connected 6-dimensional cell, and, in particular, contractible. 


\section{§3.4. Semialgebraic Structure of Teichmüller Space $T_{2}$}

The preceding argument shows that $X_{0}(\Gamma)$ can be presented as the following subset of $X(\Gamma)$ :

$$
\begin{aligned}
X_{0}(\Gamma) & =\left\{\chi \in S(\Gamma) \mid I_{\alpha_{1}}(\chi) \cdot I_{\alpha_{2}}(\chi) \cdot k(\chi)>0\right\} \\
& =\left\{\chi \in X(\Gamma) \mid I_{c_{1}}<-2 \text { and } I_{\alpha_{1}}(\chi) \cdot I_{\alpha_{2}}(\chi) \cdot k(\chi)>0\right\}
\end{aligned}
$$

where $c_{1}=\left[\alpha_{1}, \beta_{1}\right] \in \Gamma$. This presentation induces the following semialgebraic description of $X_{0}(\Gamma)$ in $X(\Gamma)$.

Theorem 3.2. $\quad X_{0}(\Gamma)$ can be described as a semialgebraic subset of $X(\Gamma)$ as follows

$$
X_{0}(\Gamma)=\left\{\chi \in X(\Gamma) \mid I_{c_{1}}(\chi)<-2, \frac{\left(I_{c_{1}}(\chi)+2\right) \cdot I_{\alpha_{1} \alpha_{2}}(\chi)}{I_{\alpha_{1}}(\chi) \cdot I_{\alpha_{2}}(\chi)}>2\right\}
$$

Hence for any representation $\rho=\left(A_{1}, B_{1}, A_{2}, B_{2}\right) \in R(\Gamma), \rho$ is a discrete and faithful $S L_{2}(\mathbf{R})$-representation of $\Gamma$ if and only if

$$
\operatorname{tr}\left(\left[A_{1}, B_{1}\right]\right)<-2 \text { and } \frac{\left(\operatorname{tr}\left(\left[A_{1}, B_{1}\right]\right)+2\right) \cdot \operatorname{tr}\left(A_{1} A_{2}\right)}{\operatorname{tr}\left(A_{1}\right) \cdot \operatorname{tr}\left(A_{2}\right)}>2 .
$$

Proof. For any $\rho=\left(A_{1}, B_{1}, A_{2}, B_{2}\right) \in t^{-1}(S(\Gamma))$, calculating $\operatorname{tr}\left(A_{1} A_{2}\right)$ gives

$$
\begin{aligned}
& k(\rho)^{2}+\left(\operatorname{tr}\left(A_{1} A_{2}\right)-\frac{2 \operatorname{tr}\left(A_{1}\right) \cdot \operatorname{tr}\left(A_{2}\right)}{\operatorname{tr}\left(\left[A_{1}, B_{1}\right]\right)+2}\right) k(\rho) \\
& \quad+\left(\frac{\operatorname{tr}\left(A_{1}\right)^{2}}{\operatorname{tr}\left(\left[A_{1}, B_{1}\right]\right)+2}-1\right)\left(\frac{\operatorname{tr}\left(A_{2}\right)^{2}}{\operatorname{tr}\left(\left[A_{1}, B_{1}\right]\right)+2}-1\right)=0 .
\end{aligned}
$$

If we consider this as a quadratic equation in $k(\rho)$, the constant term is positive. Hence the sign of $k(\rho)$ and the sign of the coefficients of the linear term of this equation are opposite each other. Thus, for $\rho=\left(A_{1}, B_{1}, A_{2}, B_{2}\right)$ $\in t^{-1}(S(\Gamma))$

$$
\operatorname{tr}\left(A_{1}\right) \cdot \operatorname{tr}\left(A_{2}\right) \cdot k(\rho)>0 \Leftrightarrow \frac{\left(\operatorname{tr}\left(\left[A_{1}, B_{1}\right]\right)+2\right) \cdot \operatorname{tr}\left(A_{1} A_{2}\right)}{\operatorname{tr}\left(A_{1}\right) \cdot \operatorname{tr}\left(A_{2}\right)}>2 .
$$


Remark. The various connected components of $X_{0}(\Gamma)$ are separated by the action of $\operatorname{Hom}(\Gamma, \mathbb{Z} / 2 \mathbb{Z})$, i.e., the sign conditions on the functions $\mathbb{I}_{\alpha_{1}}, \mathbb{I}_{\beta_{2}}, I_{\alpha_{2}}$ and $I_{\beta_{2}}$. Therefore, by adding these 4 conditions, we obtain a semialgebraic description of $T_{2}$ by means of 6 polynomial inequalities (see Corollary 2.7).

Corollary 3.2. The function $k=k(\chi)$ on $S(\Gamma)$ can be written as follows

$$
k=\frac{1}{\lambda^{4}-1} I_{\alpha_{1} \alpha_{2}}-\frac{\lambda^{2}}{\lambda^{4}-1} I_{\alpha_{1} c_{1} \alpha_{2} c_{1}^{-1}}+\frac{2}{\lambda^{2}+1} \frac{I_{\alpha_{1}} \cdot I_{\alpha_{2}}}{I_{c_{1}}+2}
$$

where $\lambda=\lambda(\chi)$ is the function on $S(\Gamma)$ defined by

$$
\lambda(\chi)+\frac{1}{\lambda(\chi)}=I_{c_{1}}(\chi)<-2 \text { and } \lambda(\chi)<-1 .
$$

Especially, the point of $T_{2}$ is completely determined by the 8 functions $\mathbb{I}_{\alpha_{1}}, \mathbb{I}_{\beta_{1}}, \mathbb{I}_{\alpha_{1} \beta_{2}}$ $(i=1,2), I_{\alpha_{1} \alpha_{2}}$ and $I_{\alpha_{1} c_{1} \alpha_{2} c_{1}^{-1}}$.

Proof. For $\rho=\left(A_{1}, B_{1}, A_{2}, B_{2}\right) \in t^{-1}(S(\Gamma))$, by comparing $\operatorname{tr}\left(A_{1} A_{2}\right)=$ $I_{\alpha_{1} \alpha_{2}}(t(\rho))$ and $\operatorname{tr}\left(A_{1}\left[A_{1}, B_{1}\right] A_{2}\left[A_{1}, B_{1}\right]^{-1}\right)=I_{\alpha_{1} c_{1} \alpha_{2} c_{1}^{-1}}(t(\rho))$, we obtain

$$
\begin{gathered}
I_{\alpha_{1} \alpha_{2}}(t(\rho))=-\frac{1}{k}\left(\frac{\lambda}{(\lambda+1)^{2}} x^{2}-1\right)\left(\frac{\lambda}{(\lambda+1)^{2}} y^{2}-1\right)-k+\frac{2 \lambda}{(\lambda+1)^{2}} x y \\
I_{\alpha_{1} c_{1} \alpha_{2} c_{1}^{-1}}(t(\rho))=-\frac{1}{\lambda^{2} k}\left(\frac{\lambda}{(\lambda+1)^{2}} x^{2}-1\right)\left(\frac{\lambda}{(\lambda+1)^{2}} y^{2}-1\right)-\lambda^{2} k+\frac{2 \lambda}{(\lambda+1)^{2}} x y
\end{gathered}
$$

where $x=\operatorname{tr}\left(A_{1}\right), \quad y=\operatorname{tr}\left(A_{2}\right), \quad \lambda<-1$ with $\lambda+\frac{1}{\lambda}=\operatorname{tr}\left(\left[A_{1}, B_{1}\right]\right)$ and $k=k(\rho)$. Subtracting $\lambda^{2}$ times the second equation from the first equation then gives the desired formula.

\section{§4. Semmialgebraic Description of Teichmïller Space $T_{g}(g \geq 3)$}

In this section, we assume $g \geq 3$. We show the connectivity and contractibility of the Teichmüller space $T_{g}$, as well as give a semialgebraic description of it, by means of arguments similar to those Section 3. 
$\S 4$.1. Definition of the Semialgebraic Subset $S(\Gamma)$ of $X(\Gamma)$

We define an open semialgebraic subset $S(\Gamma)$ of $X(\Gamma)$ by

$$
\begin{aligned}
S(\Gamma):=\left\{\chi \in X(\Gamma) \mid I_{c_{\mathrm{l}}}(\chi)\right. & <-2(i=1, \cdots, g) \\
I_{d_{j}}(\chi) & <-2(j=2, \cdots, g-2)\}
\end{aligned}
$$

where $c_{i}:=\left[\alpha_{i}, \beta_{i}\right] \in \Gamma$ and $d_{j}:=c_{1} c_{2} \cdots c_{j}$.

Similar arguments to those used to prove Propositions 3.1 and 3.2 show

Proposition 4.1. $S(\Gamma) \subset X^{\prime}(\Gamma)$. Hence $t^{-1}(S(\Gamma)) \stackrel{t}{\rightarrow} S(\Gamma)$ is a $P G L_{2}(\mathbb{R})$ bundle and can be considered as the $P G L_{2}(\mathbb{R})$-adjoint quotient of $t^{-1}(S(\Gamma))$ i.e.,

$$
S(\Gamma)=t^{-1}(S(\Gamma)) / P G L_{2}(\mathbb{R})
$$

Proposition 4.2. $X_{0}(\Gamma) \subset S(\Gamma)$.

Moreover if a representation $\rho=\left(A_{1}, B_{1}, \cdots, A_{g}, B_{g}\right)$ is contained in $R_{0}(\Gamma)$, the representation $\rho_{j}:=\left(A_{j}, B_{j}, \cdots, A_{g}, B_{g}, A_{1}, B_{1}, \cdots, A_{j-1}, B_{j-1}\right)(j=2, \cdots, g)$ is well-defined and also an element of $R_{0}(\Gamma)$, hence we have

Corollary 4.1. For $\chi \in X_{0}(\Gamma), I_{c_{1} c_{1}+1}(\chi)<-2(i=2, \cdots, g)$ where we assume that $c_{g+1}=c_{1}$.

The above arguments give rise to the following diagram.

\section{Corollary 4.2.}

$$
\begin{array}{lcccc}
R(\Gamma) \supset & R^{\prime}(\Gamma) \supset t^{-1}(S(\Gamma)) & \supset & R_{0}(\Gamma) \\
t \downarrow & \downarrow & \downarrow & \downarrow \\
X(\Gamma) \supset & X^{\prime}(\Gamma) \supset & S(\Gamma) & \supset X_{0}(\Gamma)
\end{array}
$$




\section{§4.2. Topological Structure of $S(\Gamma)$}

In this subsection, by constructing global coordinates on $S(\Gamma)$, we will show that $S(\Gamma)$ consists of $2^{2 g} \times 2^{2 g-3}$ connected components such that each component is a $6 \mathrm{~g}-6$ dimensional cell. For this purpose we need some preliminaries.

First we define a polynomial mapping $f$ from $X(\Gamma)$ to $\mathbb{R}^{3 g}$ by

$$
f(\chi):=\left(I_{\alpha_{1}}(\chi), I_{\beta_{1}}(\chi), I_{\alpha_{1} \beta_{1}}(\chi), \cdots, I_{\alpha_{g}}(\chi), I_{\beta_{g}}(\chi), I_{\alpha_{g} \beta_{g}}(\chi)\right)
$$

for $\chi \in X(\Gamma)$.

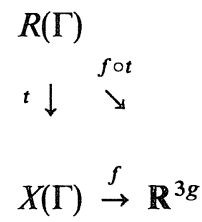

Let $\left(\vec{x}_{1}, \cdots, \vec{x}_{g}\right)$ denote the coordinates $\left(x_{11}, x_{12}, x_{13}, \cdots, x_{g 1}, x_{g 2}, x_{g 3}\right)$ on $\mathbb{R}^{3 g}$. We define a semialgebraic subset $V_{-}$by

$$
V_{-}:=\left\{\left(\vec{x}_{1}, \cdots, \vec{x}_{g}\right) \in \mathbf{R}^{3 g} \mid \kappa\left(\vec{x}_{i}\right)<-2(i=1, \cdots, g)\right\}
$$

where $\kappa(x, y, z)$ is a polynomial function on $\mathbf{R}^{3}$ defined in Subsection 3.2. Then from the definition of $S(\Gamma)$, we obtain that $f(S(\Gamma)) \subset V_{-}$. In fact we will see in the proof of Proposition 4.3 that $f(S(\Gamma))=V_{-}$.

The next lemma can be proved by the same argument as that used to prove Lemma 3.2.

Lemma 4.1. $\quad V_{-} \subset \mathbf{R}^{3 g}$ consists of $2^{2 g}$ connected components such that each component is a 3g-dimensional cell. More precisely, put

$$
U:=V_{-} \cap\left\{\left(\vec{x}_{1}, \cdots, \vec{x}_{g}\right) \in \mathbf{R}^{3 g} \mid x_{i j}>0(i=1, \cdots, g ; j=1,2)\right\}
$$

and define the action of $\mathbb{Z} / 2 \mathbb{Z})^{2 g}$ on $\mathbb{R}^{3 g}$ by multiplication by \pm 1 on the $x_{i j}$ $(i=1, \cdots, g ; j=1,2)$. Then $U$ is a $3 g$-dimensional cell and $V_{-}$can be represented as

$$
V_{-}=\coprod_{\gamma \in(\mathbf{Z} / 2 \mathbf{Z})^{2 g}} \gamma(U) \quad \text { (disjoint union). }
$$


The next lemma, which is shown by elementary calculation, is a key lemma in this section.

Lemma 4.2. 1. For a pair of hyperbolic matrices $\left(C_{1}, C_{2}\right) \in S L_{2}(\mathbf{R})^{2}$, assume that $C_{1}$ is diagonal

$$
C_{1}=\left(\begin{array}{ll}
\eta & 0 \\
0 & \frac{1}{\eta}
\end{array}\right) \quad(\eta<-1)
$$

If the traces of $C_{1}, C_{2}$ and $C_{1} C_{2}$ satisfy

$$
\left.x:=\operatorname{tr}\left(C_{1}\right)<-2, y:=\operatorname{tr}\left(C_{2}\right)<-2 \text { and } z:=\operatorname{tr}\left(C_{1} C_{2}\right)<-2 \cdots 1\right)
$$

then there exists a unique $m \in \mathbf{R}^{*}$ such that $C_{2}$ can be written as follows.

$$
C_{2}=\left(\begin{array}{cc}
\frac{\eta z-y}{\eta^{2}-1} & m \\
\frac{1}{m}\left\{\frac{\eta(\eta y-z)(\eta z-y)}{\left(\eta^{2}-1\right)^{2}}-1\right\} & \frac{\eta(\eta y-z)}{\eta^{2}-1}
\end{array}\right)
$$

Conversely, for any constant $m \in \mathbf{R}^{*}$ and $(x, y, z) \in \mathbf{R}^{3}$ with $x<-2, y<-2$ and $z<-2$, if we let $\eta$ be the unique real number $<-1$ such that $\eta+\frac{1}{\eta}=x$, and define $C_{1}=\left(\begin{array}{ll}\eta & 0 \\ 0 & \frac{1}{\eta}\end{array}\right)$ and $C_{2}$ by the condition 2), then $(x, y, z)=\left(\operatorname{tr}\left(C_{1}\right), \operatorname{tr}\left(C_{2}\right), \operatorname{tr}\left(C_{1} C_{2}\right)\right)$ as the condition 1).

2. Moreover for such a pair $\left(C_{1}, C_{2}\right) \in S L_{2}(\mathbf{R})^{2}$, we can diagonalize $C_{1} C_{2}$ and $C_{2}$ by using the following matrices $P, Q \in S L_{2}(\mathbf{R})$.

$$
P:=\left(\begin{array}{cc}
1 & -\frac{m \tau \eta}{\tau^{2}-1} \\
\frac{\tau\left(\eta^{2}-1\right)-\eta(\eta z-y)}{m \eta\left(\eta^{2}-1\right)} & \frac{\tau \eta(\eta z-y)-\left(\eta^{2}-1\right)}{\left(\eta^{2}-1\right)\left(\tau^{2}-1\right)}
\end{array}\right)
$$

where $\tau<-1$ with $\tau+\frac{1}{\tau}=z=\operatorname{tr}\left(C_{1} C_{2}\right)$ and $C_{1} C_{2}=P\left(\begin{array}{cc}\tau & 0 \\ 0 & \frac{1}{\tau}\end{array}\right) P^{-1}$. 


$$
Q:=\left(\begin{array}{cc}
1 & -\frac{m \xi}{\xi^{2}-1} \\
\frac{\xi\left(\eta^{2}-1\right)-(\eta z-y)}{m\left(\eta^{2}-1\right)} & \frac{\xi(\eta z-y)-\left(\eta^{2}-1\right)}{\left(\eta^{2}-1\right)\left(\xi^{2}-1\right)}
\end{array}\right)
$$

where $\xi<-1$ with $\xi+\frac{1}{\xi}=y=\operatorname{tr}\left(C_{2}\right)$ and $C_{2}=Q\left(\begin{array}{cc}\xi & 0 \\ 0 & \frac{1}{\xi}\end{array}\right) Q^{-1}$

In the following we write these $C_{2}, P$ and $Q$ by $C(x, y, z, m), P(x, y, z, m)$ and $Q(x, y, z, m)$.

Proposition 4.3. $S(\Gamma)$ consists of $2^{2 g} \times 2^{2 g-3}$ connected components such that each component is a $6 g-6$ dimensional cell.

Proof. We construct a mapping $\Psi$

$$
\Psi: t^{-1}(S(\Gamma)) \rightarrow V_{-} \times\{w \in \mathbb{R} \mid w<-2\}^{g-3} \times\left(\mathbb{R}^{*}\right)^{g-3} \times\left(\mathbb{R}^{*}\right)^{g} \times P G L_{2}(\mathbb{R})
$$

as follows.

For $\rho=\left(A_{1}, B_{1}, \cdots, A_{g}, B_{g}\right) \in t^{-1}(S(\Gamma))$, put

$$
\begin{aligned}
\left(\vec{x}_{1}, \cdots, \vec{x}_{g}\right) & :=f \circ t(\rho) \in V_{-}\left(\text {where } \vec{x}_{i}:=\left(x_{i 1}, x_{i 2}, x_{i 3}\right)\right) \\
C_{i} & :=\left[A_{i}, B_{i}\right](i=1, \cdots, g) \\
u_{i} & :=\operatorname{tr}\left(C_{i}\right)=\kappa\left(\vec{x}_{i}\right)(i=1, \cdots, g) \\
D_{k} & :=C_{1} \cdots C_{k}(k=1, \cdots, g-1) \\
w_{k} & :=\operatorname{tr}\left(D_{k}\right)(k=1, \cdots, g-1)
\end{aligned}
$$

We remark that

$$
\begin{gathered}
D_{1}=C_{1} \\
w_{1}=u_{1} \\
w_{g-1}=u_{g} .
\end{gathered}
$$

The definition of $S(\Gamma)$ implies that

$$
w_{1}<-2, \quad u_{2}<-2, \quad \text { and } w_{2}<-2 .
$$


Lemma 4.2.1 shows that there exists a unique $R \in P G L_{2}(\mathbf{R})$ such that

$$
\begin{aligned}
& R C_{1} R^{-1}=\left(\begin{array}{cc}
\eta_{1} & 0 \\
0 & \frac{1}{\eta_{1}}
\end{array}\right) \quad\left(\eta_{1}<-1 \text { with } \eta_{1}+\frac{1}{\eta_{1}}=w_{1}\right) \\
& R C_{2} R^{-1}=C\left(w_{1}, u_{2}, w_{2}, 1\right) .
\end{aligned}
$$

Then by Lemma 4.2 .2 there exists $P_{1}=P\left(w_{1}, u_{2}, w_{2}, 1\right)$ such that

$$
R D_{2} R^{-1}=P_{1}\left(\begin{array}{cc}
\eta_{2} & 0 \\
0 & \frac{1}{\eta_{2}}
\end{array}\right) P_{1}^{-1}\left(\eta_{2}<-1 \text { with } \eta_{2}+\frac{1}{\eta_{2}}=w_{2}\right) .
$$

Similarly because

$$
w_{2}<-2, u_{3}<-2 \text { and } w_{3}<-2
$$

Lemma 4.2.1 shows that there exists a constant $m_{1} \in \mathbf{R}^{*}$ such that

$$
R C_{3} R^{-1}=P_{1} C\left(w_{2}, u_{3}, w_{3}, m_{1}\right) P_{1}^{-1}
$$

and by Lemma 4.2 .2 there exists $P_{2}=P\left(w_{2}, u_{3}, w_{3}, m_{1}\right)$ such that

$$
R D_{3} R^{-1}=P_{1} P_{2}\left(\begin{array}{cc}
\eta_{3} & 0 \\
0 & \frac{1}{\eta_{3}}
\end{array}\right) P_{2}^{-1} P_{1}^{-1} \quad\left(\eta_{3}<-1 \text { with } \eta_{3}+\frac{1}{\eta_{3}}=w_{3}\right) .
$$

Inductively, for $j=2, \cdots, g-1$, because

$$
w_{j-1}<-2, u_{j}<-2, \text { and } w_{j}<-2
$$

Lemma 4.2 shows

$$
\begin{aligned}
& R C_{j} R^{-1}=P_{1} \cdots P_{j-2} C\left(w_{j-1}, u_{j}, w_{j}, m_{j-2}\right) P_{j-2}^{-1} \cdots P_{1}^{-1} \\
& R D_{j} R^{-1}=P_{1} \cdots P_{j-1}\left(\begin{array}{cc}
\eta_{j} & 0 \\
0 & \frac{1}{\eta_{j}}
\end{array}\right) P_{j-1}^{-1} \cdots P_{1}^{-1}
\end{aligned}
$$

where $m_{j-2} \in \mathbf{R}^{*}$ with $m_{0}=1, P_{j-1}=P\left(w_{j-1}, u_{j}, w_{j}, m_{j-2}\right)$ with $P_{0}=\left(\begin{array}{ll}1 & 0 \\ 0 & 1\end{array}\right)$ and $\eta_{j}<-1$ with $\eta_{j}+\frac{1}{\eta_{j}}=w_{j}$

Moreover, $R C_{g} R^{-1}$ can be written as 


$$
R C_{g} R^{-1}=P_{1} \cdots P_{g-2}\left(\begin{array}{cc}
0 & 1 \\
-1 & 0
\end{array}\right)\left(\begin{array}{cc}
\eta_{g-1} & 0 \\
0 & \frac{1}{\eta_{g-1}}
\end{array}\right)\left(\begin{array}{cc}
0 & -1 \\
1 & 0
\end{array}\right) P_{g-2}^{-1} \cdots P_{1}^{-1}
$$

On the other hand, by Lemma 3.3,

$$
\begin{aligned}
& R A_{1} R^{-1}=A\left(\vec{x}_{1}, k_{1}\right) \\
& R B_{1} R^{-1}=B\left(\vec{x}_{1}, k_{1}\right)
\end{aligned}
$$

for some $k_{1} \in \mathbf{R}^{*}$ where we denote $A\left(x_{11}, x_{12}, x_{13}, k_{1}\right)$ by $A\left(\vec{x}_{1}, k_{1}\right)$. By Lemma 4.2.2 there exist $Q_{2}=Q\left(w_{1}, u_{2}, w_{2}, 1\right)$ and $k_{2} \in \mathbf{R}^{*}$ such that

$$
\begin{aligned}
& R A_{2} R^{-1}=Q_{2} A\left(\vec{x}_{2}, k_{2}\right) Q_{2}^{-1} \\
& R B_{2} R^{-1}=Q_{2} B\left(\vec{x}_{2}, k_{2}\right) Q_{2}^{-1} .
\end{aligned}
$$

Inductively, for $j=2, \cdots, g-1$

$$
\begin{aligned}
& R A_{j} R^{-1}=P_{1} \cdots P_{j-2} Q_{j} A\left(\vec{x}_{j}, k_{j}\right) Q_{j}^{-1} P_{j-2}^{-1} \cdots P_{1}^{-1} \\
& R B_{j} R^{-1}=P_{1} \cdots P_{j-2} Q_{j} B\left(\vec{x}_{j}, k_{j}\right) Q_{j}^{-1} P_{j-2}^{-1} \cdots P_{1}^{-1}
\end{aligned}
$$

where $Q_{j}=Q\left(w_{j-1}, u_{j}, w_{j}, m_{j-2}\right)$ and $k_{j} \in \mathbf{R}^{*}$. Moreover

$$
\begin{aligned}
& R A_{g} R^{-1}=P_{1} \cdots P_{g-2}\left(\begin{array}{cc}
0 & 1 \\
-1 & 0
\end{array}\right) A\left(\vec{x}_{g}, k_{g}\right)\left(\begin{array}{cc}
0 & -1 \\
1 & 0
\end{array}\right) P_{g-2}^{-1} \cdots P_{1}^{-1} \\
& R B_{g} R^{-1}=P_{1} \cdots P_{g-2}\left(\begin{array}{cc}
0 & 1 \\
-1 & 0
\end{array}\right) B\left(\vec{x}_{g}, k_{g}\right)\left(\begin{array}{cc}
0 & -1 \\
1 & 0
\end{array}\right) P_{g-2}^{-1} \cdots P_{1}^{-1}
\end{aligned}
$$

for some $k_{g} \in \mathbf{R}^{*}$. Now we can define the mapping $\Psi$

$$
\begin{aligned}
t^{-1}(S(\Gamma)) & \stackrel{\Psi}{\rightarrow} V_{-} \times\{w \in \mathbb{R} \mid w<-2\}^{g-3} \times\left(\mathbf{R}^{*}\right)^{g-3} \times\left(\mathbb{R}^{*}\right)^{g} \times P G L_{2}(\mathbb{R}) \\
\rho & \mapsto\left(f \circ t(\rho), w_{2}, \cdots, w_{g-2}, m_{1}, \cdots, m_{g-3}, k_{1}, \cdots, k_{g}, R\right) .
\end{aligned}
$$

Lemma 4.2 shows that this mapping is bijective and homeomorphic. $\Psi$ induces a homeomorphism $\Phi$ as follows 


$$
\begin{gathered}
t^{-1}(S(\Gamma)) \stackrel{\Psi}{\simeq} V_{-} \times\{w \in \mathbf{R} \mid w<-2\}^{g-3} \times\left(\mathbf{R}^{*}\right)^{g-3} \times\left(\mathbf{R}^{*}\right)^{g} \times P G L_{2}(\mathbf{R}) \\
\quad \downarrow \\
\downarrow \\
\quad \downarrow^{\text {proj. }} \\
\qquad(\Gamma) \stackrel{\Phi}{\rightarrow} \quad V_{-} \times\{w \in \mathbf{R} \mid w<-2\}^{g-3} \times\left(\mathbf{R}^{*}\right)^{g-3} \times\left(\mathbf{R}^{*}\right)^{g} .
\end{gathered}
$$

Thus by Lemma $4.1, S(\Gamma)$ consists of $2^{2 g} \times 2^{2 g-3}$ connected components such that each component is a $6 \mathrm{~g}-6$ dimensional cell.

\section{§4.3. Cell Structure of Teichmüller Space $T_{g}$}

In the following, by using the global coordinate functions on $S(\Gamma)$ constructed in the previous subsection, we give conditions which characterize the connected components of $X_{0}(\Gamma)$ in $S(\Gamma)$.

Proposition 4.4. On $X_{0}(\Gamma)$, the component $m_{j}(j=1, \cdots, g-3)$ of the mapping $\Phi$ is positive.

This is equivalent to the next proposition for the space of representations.

Proposition 4.5. For $\rho=\left(A_{1}, B_{1}, \cdots, A_{g}, B_{g}\right) \in R_{0}(\Gamma)$, the value $m_{j}(\rho)$ of the component $m_{j}(j=1, \cdots, g-3)$ of the mapping $\Psi$ at $\rho$ is positive.

To prove this, we need the following elementary lemma.

Lemma 4.3. If the matrices $P, C(m) \in S L_{2}(\mathbf{R})(m<0)$ satisfy

$$
\begin{aligned}
P & =\left(\begin{array}{ll}
a & b \\
c & d
\end{array}\right)(a>0, b<0, c<0, d>0) \cdots(\star) \\
C(m) & =\left(\begin{array}{cc}
x & m \\
\frac{1}{m}(x w-1) & w
\end{array}\right) \quad(x>0, w<0, m<0, x+w<-2)
\end{aligned}
$$

then there exists $m_{0}<0$ such that $P C\left(m_{0}\right) P^{-1}$ is a lower triangular matrix. Moreover if $P_{1}, P_{2} \in S L_{2}(\mathbf{R})$ satisfy the above condition $(\star)$, then $P_{1} P_{2}$ also satisfies the above condition $(\star)$.

Proof of Proposition 4.5. We use the notations of the proof of Proposition 4.3. We apply the above Lemma 4.3 to $P_{1}$ and $C\left(w_{2}, u_{3}, w_{3}, m_{1}\right)$ to conclude 
that $m_{1}>0$ on $R_{0}(\Gamma)$ because $\rho(\Gamma)$ is a discrete purely hyperbolic subgrourp of $S L_{2}(\mathbb{R}), R C_{1} R^{-1}$ and $R C_{3} R^{-1}$ cannot have a common fixed point. Hence $P_{2}$ and $P_{1} P_{2}$ also satisfy the condition (*) in Lemma 4.3 and by applying it to $P_{1} P_{2}$ and $C\left(w_{3}, u_{4}, w_{4}, m_{2}\right)$, we get $m_{2}>0$ on $R_{0}(\Gamma)$. Successive repetition of this procedure proves the Proposition.

Proposition 4.6. On $X_{0}(\Gamma)$, the product of components $x_{i 1} \cdot k_{i}$ of the mapping $\Phi$ is positive $(i=1, \cdots, g)$.

This is equivalent to the next proposition for the space of representations.

Proposition 4.7. For $\rho=\left(A_{1}, B_{1}, \cdots, A_{g}, B_{g}\right) \in R_{0}(\Gamma)$, the value $x_{i 1}(\rho) \cdot k_{i}(\rho)$ of the product of components $x_{i 1}$ and $k_{i}$ of the mapping $\Psi$ at $\rho$ is positive $(i=1, \cdots, g)$.

Proof. We use the notations in the proof of Proposition 4.3. For $\rho=\left(A_{1}, B_{1}, \cdots, A_{g}, B_{g}\right) \in R_{0}(\Gamma)$, a computation shows that

$$
\begin{aligned}
\operatorname{tr}\left(A_{1} C_{2}\right)= & \operatorname{tr}\left(R A_{1} R^{-1} R C_{2} R^{-1}\right) \\
= & \operatorname{tr}\left(A\left(\vec{x}_{1}, k_{1}\right) C\left(w_{1}, u_{2}, w_{2}, 1\right)\right) \\
= & \frac{1}{k_{1}}\left(\frac{x_{11}^{2}}{w_{1}+2}-1\right)\left\{\frac{\eta_{1}\left(\eta_{1} u_{2}-w_{2}\right)\left(\eta_{1} w_{2}-u_{2}\right)}{\left(\eta_{1}^{2}-1\right)^{2}}-1\right\} \\
& +k_{1}+\frac{x_{11}\left(w_{2}+u_{2}\right)}{w_{1}+2} .
\end{aligned}
$$

Suppose that there exists a connected component $U$ of $R_{0}(\Pi)$ such that $U$ contains a representation $\rho$ with $x_{11} \cdot k_{1}<0$. Then the function $x_{11} \cdot k_{1}$ is negative on $U$. Moreover there exist $t<-2$ and $\rho \in U$ such that

$$
-\left|x_{11}\right|=w_{1}=w_{2}=u_{2}=t \text { and }\left|k_{1}\right|=-\frac{t^{2}}{t+2}+1 .
$$

Then since $x_{11} \cdot k_{1}<0$

$$
\begin{aligned}
\operatorname{tr}\left(A_{1} C_{2}\right) & = \pm \frac{2 t^{2}}{t+2} \mp 2\left(\frac{t^{2}}{t+2}-1\right) \\
& = \pm 2
\end{aligned}
$$

This contradicts the pure hyperbolicity of $\rho \in R_{0}(\Gamma)$ (see Proposition 2.3). Hence 
$x_{11} \cdot k_{1}$ is positive on $R_{0}(\Gamma)$.

Next we will show that the function $x_{j 1} \cdot k_{j}(j=2, \cdots, g)$ is also positive on $R_{0}(\Gamma)$. For $\rho=\left(A_{1}, B_{1}, \cdots, A_{g}, B_{g}\right) \in R_{0}(\Gamma)$, Proposition 4.2 and Corollary 4.1 show that

$$
\operatorname{tr} C_{j}<-2, \quad \operatorname{tr} C_{j+1}<-2 \text { and } \operatorname{tr}\left(C_{j} C_{j+1}\right)<-2(j=1, \cdots, g) .
$$

Hence by Lemma 4.2 there exists a unique $R_{j} \in P G L_{2}(\mathbf{R})$ such that

$$
\begin{aligned}
R_{j} C_{j} R_{j}^{-1} & =\left(\begin{array}{cc}
\lambda_{j} & 0 \\
0 & \frac{1}{\lambda_{j}}
\end{array}\right) \\
R_{j} C_{j+1} R_{j}^{-1} & =C\left(\operatorname{tr} C_{j}, \operatorname{tr} C_{j+1}, \operatorname{tr}\left(C_{j} C_{j+1}\right), 1\right)
\end{aligned}
$$

where $\lambda_{j}<-1$ with $\lambda_{j}+\frac{1}{\lambda_{j}}=\operatorname{tr} C_{j}(j=1, \cdots, g)$. We remark that $R_{1}=R$ and $\lambda_{1}=\eta_{1}$. Then by Lemma 3.3 there exists $k_{j} \in \mathbb{R}^{*}(j=1, \cdots, g)$ such that

$$
R_{j} A_{j} R_{j}^{-1}=A\left(\vec{x}_{j}, \hat{k}_{j}\right)
$$

We remark that $k_{1}=k_{1}$. Moreover, the argument just applied to show that $x_{11} \cdot k_{1}>0$ on $R_{0}(\Gamma)$ also implies (by permuting the A's and B's as in the paragraph preceding Corollary 4.1) that $x_{j 1} \cdot \hat{k}_{j}>0$ on $R_{0}(\Gamma)(j=1, \cdots, g)$. In the following, we will show that as functions on $R_{0}(\Gamma), k_{j}$ and $k_{j}$ have the same signs on each connected component of $R_{0}(\Gamma)$. More precisely, for $\rho \in R_{0}(\Gamma)$, there exists $s_{j} \in \mathbb{R}^{*}(j=1, \cdots, g)$ such that $s_{j}^{2} \cdot k_{j}=k_{j}$. By proving this claim, we will thus obtain that $x_{j 1} \cdot k_{j}>0$ on $R_{0}(\Gamma)$ is equivalent to $x_{j 1} \cdot k_{j}>0$ on $R_{0}(\Gamma)$. This will complete our proof.

For $j=2, \cdots, g-2$ put

$$
\hat{R}_{j}:=Q_{j}^{-1} P_{j-2}^{-1} \cdots P_{1}^{-1} R
$$

Then we have

$$
\begin{aligned}
\hat{R}_{j} C_{j} \hat{R}_{j}^{-1} & =\left(\begin{array}{cc}
\lambda_{j} & 0 \\
0 & \frac{1}{\lambda_{j}}
\end{array}\right) \\
\hat{R}_{j} C_{j+1} \hat{R}_{j}^{-1} & =Q_{j}^{-1} P_{j-1} C\left(w_{j}, u_{j+1}, w_{j+1}, m_{j-1}\right) P_{j-1}^{-1} Q_{j} \\
\hat{R}_{j} A_{j} \hat{R}_{j}^{-1} & =A\left(\vec{x}_{j}, k_{j}\right) .
\end{aligned}
$$

Proposition 4.5 implies that $m_{j}>0$ on $R_{0}(\Gamma)(j=1, \cdots, g-3)$. Hence the signs of entries of the matrices $C\left(w_{j}, u_{j+1}, w_{j+1}, m_{j-1}\right), P_{j-1}$ and $Q_{j}$ are $\left(\begin{array}{c}+ \\ -\end{array}\right)$, 
$\left(\begin{array}{ll}+ & - \\ - & +\end{array}\right)$ and $\left(\begin{array}{ll}+ & + \\ - & -\end{array}\right)$. Therefore there exists $s_{j} \in \mathbb{R}^{*}$ such that

$$
\begin{aligned}
\left(\begin{array}{cc}
s_{j} & 0 \\
0 & \frac{1}{s_{j}}
\end{array}\right) \hat{R}_{j} C_{j} \hat{R}_{j}^{-1}\left(\begin{array}{cc}
\frac{1}{s_{j}} & 0 \\
0 & s_{j}
\end{array}\right) & =\left(\begin{array}{cc}
\lambda_{j} & 0 \\
0 & \frac{1}{\lambda_{j}}
\end{array}\right) \\
\left(\begin{array}{cc}
s_{j} & 0 \\
0 & \frac{1}{s_{j}}
\end{array}\right) \hat{R}_{j} C_{j+1} \hat{R}_{j}^{-1}\left(\begin{array}{cc}
\frac{1}{s_{j}} & 0 \\
0 & s_{j}
\end{array}\right) & =C\left(\operatorname{tr} C_{j}, \operatorname{tr} C_{j+1}, \operatorname{tr}\left(C_{j} C_{j+1}\right), 1\right) \\
\left(\begin{array}{cc}
s_{j} & 0 \\
0 & \frac{1}{s_{j}}
\end{array}\right) \hat{R}_{j} A_{j} \hat{R}_{j}^{-1}\left(\begin{array}{cc}
\frac{1}{s_{j}} & 0 \\
0 & s_{j}
\end{array}\right) & =A\left(\vec{x}_{j}, s_{j}^{-2} k_{j}\right) .
\end{aligned}
$$

Hence

$$
R_{j}=\left(\begin{array}{cc}
s_{j} & 0 \\
0 & \frac{1}{s_{j}}
\end{array}\right) \hat{R}_{j} \text { and } k_{j}=s_{j}^{2} \hat{k}_{j}
$$

Similarly put

$$
\begin{aligned}
\hat{R}_{g-1} & =Q_{g-1}^{-1} P_{g-3}^{-1} \cdots P_{1}^{-1} R \\
\hat{R}_{g} & =\left(\begin{array}{cc}
0 & -1 \\
1 & 0
\end{array}\right) P_{g-2}^{-1} \cdots P_{1}^{-1} R
\end{aligned}
$$

Then a similar argument shows that there exist $s_{g-1}, s_{g} \in \mathbf{R}^{*}$ such that

$$
\begin{aligned}
R_{g-1} & =\left(\begin{array}{cc}
s_{g-1} & 0 \\
0 & \frac{1}{s_{g-1}}
\end{array}\right) \hat{R}_{g-1} \text { and } k_{g-1}=s_{g-1}^{2} \hat{k}_{g-1} \\
R_{g} & =\left(\begin{array}{cc}
s_{g} & 0 \\
0 & \frac{1}{s_{g}}
\end{array}\right) \hat{R}_{g} \text { and } k_{g}=s_{g}^{2} \hat{k}_{g} .
\end{aligned}
$$

The above arguments show that

$$
X_{0}(\Gamma) \subset\left\{\chi \in S(\Gamma) \mid m_{j}>0(j=1, \cdots, g-3), x_{i 1} k_{i}>0(i=1, \cdots, g)\right\} .
$$

Then by considering the number of connected components of $X_{0}(\Gamma)$, we see that $\pi_{0}\left(X_{0}(\Gamma)\right)$ is less than or equal to $2^{2 g}$. On the other hand we have seen in Subsection 2.4 that $\pi_{0}\left(X_{0}(\Gamma)\right) \geq 2^{2 g}$. Hence we get the following result.

Theorem 4.1. $\pi_{0}\left(X_{0}(\Gamma)\right)=2^{2 g}$. Thus 


$$
T_{g}=\operatorname{Hom}(\Gamma, \mathbf{Z} / 2 \mathbb{Z}) \backslash X_{0}(\Gamma)
$$

is a connected (6g-6)-dimensional cell, and, in particular, contractible.

\section{§4.4. Semialgebraic Structure of Teichmüller Space $T_{g}$}

Now $X_{0}(\Gamma)$ can be written as

$$
X_{0}(\Gamma)=\left\{\chi \in S(\Gamma) \mid m_{j}>0(j=1, \cdots, g-3), x_{i 1} k_{i}>0(i=1, \cdots, g)\right\} .
$$

In the following we will rewrite the above presentation of $X_{0}(\Gamma)$ by using polynomial inequalities in the $I_{h}(h \in \Gamma)$.

Proposition 4.8. For a representation $\rho=\left(A_{1}, B_{1}, \cdots, A_{g}, B_{g}\right) \in t^{-1}(S(\Gamma))$ we denote by $m_{j}(\rho)(j=1, \cdots, g-3)$ by $m_{j}$ for the sake of simplicity. Then

$$
m_{j}>0(j=1, \cdots, g-3)
$$

if and only if

$$
\begin{aligned}
& \operatorname{tr} D_{j+1}\left(\operatorname{tr} D_{j} \operatorname{tr} D_{j+2}+\operatorname{tr} C_{j+1} \operatorname{tr} C_{j+2}\right)-2\left(\operatorname{tr} D_{j} \operatorname{tr} C_{j+2}+\operatorname{tr} C_{j+1} \operatorname{tr} D_{j+2}\right) \\
& \quad>\left\{\left(\operatorname{tr} D_{j+1}\right)^{2}-4\right\} \operatorname{tr}\left(D_{j} C_{j+2}\right)(j=1, \cdots, g-3)
\end{aligned}
$$

where $C_{i}:=\left[A_{i}, B_{i}\right](i=1, \cdots, g)$ and $D_{j}:=C_{1} \cdots C_{j}(j=1, \cdots, g-1)$.

Proof. We use the notations of the proof of Proposition 4.3. We calculate $\operatorname{tr}\left(D_{j} C_{j+2}\right)(j=1, \cdots, g-3)$ for $\rho=\left(A_{1}, B_{1}, \cdots, A_{g}, B_{g}\right) \in t^{-1}(S(\Gamma))$.

$$
\begin{aligned}
\operatorname{tr}\left(D_{j} C_{j+2}\right)= & \operatorname{tr}\left(R D_{j} R^{-1} R C_{j+2} R^{-1}\right) \\
= & \operatorname{tr}\left(P_{1} \cdots P_{j-1}\left(\begin{array}{cc}
\eta_{j} & 0 \\
0 & \frac{1}{\eta_{j}}
\end{array}\right) P_{j-1}^{-1} \cdots P_{1}^{-1}\right. \\
& \left.\times P_{1} \cdots P_{j} C\left(w_{j+1}, u_{j+2}, w_{j+2}, m_{j}\right) P_{j}^{-1} \cdots P_{1}^{-1}\right) \\
= & \operatorname{tr}\left(P_{j}^{-1}\left(\begin{array}{cc}
\eta_{j} & 0 \\
0 & \frac{1}{\eta_{j}}
\end{array}\right) P_{j} C\left(w_{j+1}, u_{j+2}, w_{j+2}, m_{j}\right)\right) \\
= & \frac{w_{j+1}\left(w_{j} w_{j+2}+u_{j+1} u_{j+2}\right)-2\left(w_{j} u_{j+2}+u_{j+1} w_{j+2}\right)}{w_{j+1}^{2}-4} \\
& +\frac{m_{j-1}}{m_{j}} \cdot \frac{-\eta_{j+1}-\eta_{j+1}^{3} \eta_{j}^{2}+\eta_{j+1}^{2} \eta_{j} u_{j+1}}{\left(\eta_{j+1}^{2}-1\right)^{2}}
\end{aligned}
$$




$$
\begin{aligned}
& \times\left\{\frac{\eta_{j+1}\left(\eta_{j+1} u_{j+2}-w_{j+2}\right)\left(\eta_{j+1} w_{j+2}-u_{j+2}\right)}{\left(\eta_{j+1}^{2}-1\right)^{2}}-1\right\} \\
& +\frac{m_{j}}{m_{j-1}} \cdot \frac{\eta_{j}^{2} \eta_{j+1}^{-1}+\eta_{j+1}-\eta_{j} u_{j+1}}{\eta_{j}^{2}} .
\end{aligned}
$$

Hence we get the following quadratic equation in $\frac{m_{j}}{m_{j-1}}$

$$
\begin{aligned}
& \left(\frac{\eta_{j}^{2} \eta_{j+1}^{-1}+\eta_{j+1}-\eta_{j} u_{j+1}}{\eta_{j}^{2}}\right) \cdot\left(\frac{m_{j}}{m_{j-1}}\right)^{2} \\
& \quad+\left\{\frac{w_{j+1}\left(w_{j} w_{j+2}+u_{j+1} u_{j+2}\right)-2\left(w_{j} u_{j+2}+u_{j+1} w_{j+2}\right)}{w_{j+1}^{2}-4}-\operatorname{tr}\left(D_{j} C_{j+2}\right)\right\} \\
& \quad \times\left(\frac{m_{j}}{m_{j-1}}\right)+\left(\frac{-\eta_{j+1}-\eta_{j+1}^{3} \eta_{j}^{2}+\eta_{j+1}^{2} \eta_{j} u_{j+1}}{\left(\eta_{j+1}^{2}-1\right)^{2}}\right) \\
& \quad \times\left\{\frac{\eta_{j+1}\left(\eta_{j+1} u_{j+2}-w_{j+2}\right)\left(\eta_{j+1} w_{j+2}-u_{j+2}\right)}{\left(\eta_{j+1}^{2}-1\right)^{2}}-1\right\} \\
& =0
\end{aligned}
$$

One can easily check that the coefficients of $\left(\frac{m_{J}}{m_{J}-1}\right)^{2}$ and constant terms are negative. Hence if we put $m_{0}=1$, then

$$
\begin{aligned}
& m_{j}>0(j=1, \cdots, g-3) \\
\Leftrightarrow & \frac{m_{j}}{m_{j-1}}>0 \quad(j=1, \cdots, g-3) \\
\Leftrightarrow & \frac{w_{j+1}\left(w_{j} w_{j+2}+u_{j+1} u_{j+2}\right)-2\left(w_{j} u_{j+2}+u_{j+1} w_{j+2}\right)}{w_{j+1}^{2}-4}-\operatorname{tr}\left(D_{j} C_{j+2}\right)>0 \\
& \quad(j=1, \cdots, g-3)
\end{aligned}
$$

Since $w_{j+1}^{2}-4>0$, we have prove our assertion.

We put

$$
S^{\prime}(\Gamma):=\left\{\chi \in S(\Gamma) \mid m_{j}(\chi)>0(j=1, \cdots, g-3)\right\}
$$

Proposition 4.9. For $\rho=\left(A_{1}, B_{1}, \cdots, A_{g}, B_{g}\right) \in t^{-1}\left(S^{\prime}(\Gamma)\right)$ we write $x_{i 1}(\rho) \cdot k_{i}(\rho)$ $(i=1, \cdots, g)$ by $x_{i 1} \cdot k_{i}$ for the sake of simplicity. Then 


$$
x_{i 1} \cdot k_{i}>0(i=1, \cdots, g)
$$

if and only if

$$
\frac{\operatorname{tr}\left(\left[A_{i}, B_{i}\right]\left[A_{i+1}, B_{i+1}\right]\right)+\operatorname{tr}\left[A_{i+1}, B_{i+1}\right]}{\operatorname{tr}\left[A_{i}, B_{i}\right]+2}<\frac{\operatorname{tr}\left(A_{i}\left[A_{i+1}, B_{i+1}\right]\right)}{\operatorname{tr} A_{i}} .
$$

Proof. We use the notations of the proof of Proposition 4.7. We compute $\operatorname{tr}\left(A_{i} C_{i+1}\right)(i=1, \cdots, g)$ for $\rho=\left(A_{1}, B_{1}, \cdots, A_{g}, B_{g}\right) \in t^{-1}\left(S^{\prime}(\Gamma)\right)$.

$$
\begin{aligned}
\operatorname{tr}\left(A_{i} C_{i+1}\right)= & \operatorname{tr}\left(R_{i} A_{i} R_{i}^{-1} R_{i} C_{i+1} R_{i}^{-1}\right) \\
= & \operatorname{tr}\left(A\left(\vec{x}_{i}, \hat{k}_{i}\right) C\left(\operatorname{tr} C_{i}, \operatorname{tr} C_{i+1}, \operatorname{tr}\left(C_{i} C_{i+1}\right), 1\right)\right) \\
= & \hat{k}_{i}+\frac{\operatorname{tr} A_{i} \cdot\left(\operatorname{tr}\left(C_{i} C_{i+1}\right)+\operatorname{tr} C_{i+1}\right)}{\operatorname{tr} C_{i}+2}+\frac{1}{\hat{k}_{i}}\left(\frac{\left(\operatorname{tr} A_{i}\right)^{2}}{\operatorname{tr} C_{i}+2}-1\right) \\
& \times\left\{\frac{\left(\lambda_{i} \operatorname{tr} C_{i+1}-\operatorname{tr}\left(C_{i} C_{i+1}\right)\right)\left(\lambda_{i} \operatorname{tr}\left(C_{i} C_{i+1}\right)-\operatorname{tr} C_{i+1}\right)}{\left(\operatorname{tr} C_{i}+2\right)\left(\lambda_{i}-1\right)^{2}}-1\right\} .
\end{aligned}
$$

Hence we get the following quadratic equation in $k_{i}$.

$$
\begin{aligned}
& \hat{k}_{i}^{2}+\left\{\frac{\operatorname{tr} A_{i} \cdot\left(\operatorname{tr}\left(C_{i} C_{i+1}\right)+\operatorname{tr} C_{i+1}\right)}{\operatorname{tr} C_{i}+2}-\operatorname{tr}\left(A_{i} C_{i+1}\right)\right\} \hat{k}_{i} \\
& +\left(\frac{\left(\operatorname{tr} A_{i}\right)^{2}}{\operatorname{tr} C_{i}+2}-1\right)\left\{\frac{\left(\lambda_{i} \operatorname{tr} C_{i+1}-\operatorname{tr}\left(C_{i} C_{i+1}\right)\right)\left(\lambda_{i} \operatorname{tr}\left(C_{i} C_{i+1}\right)-\operatorname{tr}\left(C_{i+1}\right)\right.}{\left(\operatorname{tr} C_{i}+2\right)\left(\lambda_{i}-1\right)^{2}}-1\right\} \\
& =0
\end{aligned}
$$

As the constant term is positive

$$
\begin{aligned}
& x_{i 1} \cdot k_{i}>0(i=1, \cdots, g) \\
\Leftrightarrow & x_{i 1} \cdot \hat{k}_{i}>0(i=1, \cdots, g) \\
\Leftrightarrow & \frac{\operatorname{tr}\left(C_{i} C_{i+1}\right)+\operatorname{tr} C_{i+1}}{\operatorname{tr} C_{i}+2}<\frac{\operatorname{tr}\left(A_{i} C_{i+1}\right)}{\operatorname{tr} A_{i}}(i=1, \cdots, g) .
\end{aligned}
$$

The above considerations give rise to the following semialgebraic presentation of $X_{0}(\Gamma)$.

Theorem 4.2. For $\alpha_{i}, \beta_{i} \in \Gamma$, put $c_{i}:=\left[\alpha_{i}, \beta_{i}\right](i=1, \cdots, g)$, and $d_{j}:=c_{1} \cdots c_{j}$ 
$(j=1, \cdots, g-1)$. Then $\chi \in X(\Gamma)$ is contained in $X_{0}(\Gamma)$ if and only if $\chi$ satisfies the following $4 g-6$ inequalities on $I_{h}(\in \Gamma)$.

$$
\begin{aligned}
& I_{c_{l}}(\chi)<-2(i=1, \cdots, g), \\
& I_{d_{j}}(\chi)<-2(j=2, \cdots, g-2), \\
& \frac{I_{c_{k} c_{k+1}}(\chi)+I_{c_{k+1}}(\chi)}{I_{c_{k}}(\chi)+2}<\frac{I_{\alpha_{k} c_{k+1}}(\chi)}{I_{\alpha_{k}}(\chi)}(k=1, \cdots, g), \\
& I_{d_{l+1}}(\chi)\left(I_{d_{l}}(\chi) I_{d_{l+2}}(\chi)+I_{c_{l+1}}(\chi) I_{c_{l+2}}(\chi)\right) \\
& \quad>2\left(I_{d_{l}}(\chi) I_{c_{l+2}}(\chi)+I_{c_{l+1}}(\chi) I_{d_{l+2}}(\chi)\right)+\left(I_{d_{l+1}}(\chi)^{2}-4\right) I_{d_{l} c_{l+2}}(\chi) \\
& \quad(l=1, \cdots, g-3)
\end{aligned}
$$

where $c_{g+1}=c_{1}$.

Hence by adding $2 g$ inequalities which consist of the sign conditions of $I_{\alpha_{1}}$, $I_{\beta_{1}}(i=1, \cdots, g)$, we can also describe $T_{g}$ by $6 g-6$ polynomial inequalities in $X(\Gamma)$.

\section{References}

[B-C-R] Bochnak, J., Coste, M. and Roy, M-F., Géométrie algébrique réelle, Springer Verlag, 1986.

[Br] Brumfiel, G. W., The real spectrum compactification of Teichmüller space, Contem. Math., 74 (1988), 51-75.

[C-S] Culler, M. and Shalen, P. B., Varieties of group representations and splitting of 3 manifolds, Ann. of Math., 117 (1983), 109-146.

[F] Fricke, R. and Klein, F., Vorlesungen uber der Theorie der Automorphen Funktionen, 1, Teubner, Leipzig. 1897

[Go] Goldman, W., Topological components of spaces of representations, Invent Math., 93 (1988), 557-607.

[Gu] Gunning, R. C., Lectures on vector bundles over Riemann surfaces, Princeton, 1967.

[He] Helling, H., Diskrete Untergruppen von $S L_{2}(\mathbf{R})$, Invent. Math., 17 (1972), 217-229.

[Ho] Horowitz, R., Characters of free groups represented in the two dimensional linear groups, Comm. Pure Appl. Math., 25 (1972), 635-649.

[Jø] Jørgensen, T., On discrete groups of Möbius transformations, Amer. J. Math., 98 (1976), 739-749.

[K1] Keen, L., Intrinsic moduli, Ann. of Math., 84 (1966), 404-420.

[K2] - On Fricke moduli, Ann. of Math. Stud. 66 (1971), 205-224.

[K3] - A correction to "On Fricke moduli", Proc. Amer. Math. Soc., 40 (1973), 60-62.

[M-S] Morgan, J. W. and Shalen, P. B., Valuations, trees and degenerations of hyperbolic structures, Ann. of Math., 120 (1984), 401-476.

[Ok] Okai, T., An explicit description of the Teichmüller space as holonomy representations and its applications, Hiroshima Math. J., 22 (1992), 259-271.

[O1] Okumura, Y., On the global real analytic coordinates for Teichmüller spaces, J. Math. Soc. Japan, 42, No. 1 (1990), 91-101.

[O2] - On lifting problem of Kleinian groups into $S L(2, \mathrm{C})$, Summer Seminar on Function Theory, July 1992. 
[Pa] Patterson, S. J., On the cohomology of Fuchsian groups, Glasgow' Math., 16 (1975), 123-140.

[Pr] Procesi, C., The invariant theory of $n \times n$ matrices, Adv. in Math., 19 (1976), 306-381.

[Sa 1] Saito, K., The Teichmüller space and a certain modular function from a view point of group representation, Algebraic Geometry and Related Topics-Proceedings of the International Symposium, Inchoen, Republic of Korea (1992), 41-89.

[Sa 2] Representation varieties of a finitely generated group into $S L_{2}$ or $G L_{2}$, RIMS-preprint, 958 (December 1993).

[Sa 3$]$ Algebraic representation of Teichmüller space, Kodai Math. J., 17 (1994), 609-626.

[S-S] Seppälä, M. and Sorvali, T., Traces of commutators of Möbius transformations, Math. Scand., 68 (1991), 53-58.

[Si] Siegel, C. L., Topics in complex function theory, 2, John Wiley and Sons, Inc. 1971.

[W] Weil, A., On discrete subgroups of Lie groups, Ann. of Math., 72 (1960), 369-384, Ann. of Math., 75 (1962), 578-602. 
